\title{
A canonical rate-independent model of geometrically linear isotropic gradient plasticity with isotropic hardening and plastic spin accounting for the Burgers vector
}

\author{
François Ebobisse ${ }^{1}$ and Klaus Hackl ${ }^{2}$ and Patrizio Neff ${ }^{3}$
}

April 8, 2019

\begin{abstract}
In this paper we propose a canonical variational framework for rate-independent phenomenological geometrically linear gradient plasticity with plastic spin. The model combines the additive decomposition of the total distortion into non-symmetric elastic and plastic distortions, with a defect energy contribution taking account of the Burgers vector through a dependence only on the dislocation density tensor Curl $p$ giving rise to a non-symmetric nonlocal backstress, and isotropic hardening response only depending on the accumulated equivalent plastic strain. The model is fully isotropic and satisfies linearized gauge-invariance conditions, i.e., only true state-variables appear. The model satisfies also the principle of maximum dissipation which allows to show existence for the weak formulation. For this result, a recently introduced Korn's inequality for incompatible tensor fields is necessary. Uniqueness is shown in the class of strong solutions. For vanishing energetic length scale, the model reduces to classical elasto-plasticity with symmetric plastic strain $\varepsilon_{p}$ and standard isotropic hardening.
\end{abstract}

Key words: plasticity, gradient plasticity, variational modeling, dissipation function, geometrically necessary dislocations, incompatible distortions, rate-independent models, isotropic hardening, generalized standard material, variational inequality, convex analysis, associated flow rule, defect energy, dislocation density, plastic rotation, global dissipation inequality, Burgers vector, plastic spin.

AMS 2010 subject classification: 35D30, 35D35, 74C05, 74C15, 74D10, 35J25.

\footnotetext{
${ }^{1}$ Corresponding author, François Ebobisse, Department of Mathematics and Applied Mathematics, University of Cape Town, Rondebosch 7700, South Africa, e-mail: francois.ebobissebille@uct.ac.za

${ }^{2}$ Klaus Hackl, Lehrstuhl für Mechanik-Materialtheorie, Ruhr-Universität Bochum, Universitätsstrasse 150, 44801 Bochum, Germany, e-mail: klaus.hackl@rub.de

${ }^{3}$ Patrizio Neff, Lehrstuhl für Nichtlineare Analysis und Modellierung, Fakultät für Mathematik, Universität Duisburg-Essen, Thea-Leymann Str. 9, 45127 Essen, Germany, e-mail: patrizio.neff@uni-due.de, http://www.unidue.de/mathematik/ag_neff
} 


\section{Contents}

1 Introduction $\quad 2$

2 Some notational agreements and definitions $\quad 6$

3 The description of the model 8

3.1 The balance equation . . . . . . . . . . . . . . . . . . . . . . 8

3.2 Constitutive equations. . . . . . . . . . . . . . . . . . . . . . . . 9

3.2.1 The derivation of the dissipation inequality . . . . . . . . . . . . . . . . 9

3.2.2 The boundary conditions on the plastic distortion . . . . . . . . . . . . . . 11

3.3 The flow rule . . . . . . . . . . . . . . . . . . . . . . . . . 11

3.3.1 The flow rule in its primal formulation . . . . . . . . . . . . . . . . . . 11

3.3.2 The flow rule in its dual formulation . . . . . . . . . . . . . . . . . . . 13

4 The complete mathematical formulation in the case $\alpha_{2}>0 \quad 16$

4.1 The strong formulation . . . . . . . . . . . . . . . . . . . . . . 16

4.2 The weak formulation . . . . . . . . . . . . . . . . . . . . . . 18

4.3 Existence result for the weak formulation . . . . . . . . . . . . . . . . . . 18

4.4 Uniqueness of the strong solution . . . . . . . . . . . . . . . . . . . . . . 21

4.5 Perfect gradient plasticity with spin . . . . . . . . . . . . . . . . . . . 23

5 The complete mathematical formulation in the no-spin-hardening case 24

5.1 The strong formulation of the model . . . . . . . . . . . . . . . . . . . . . . 24

5.2 The weak formulation of the model . . . . . . . . . . . . . . . . . . . . . . . . 24

5.3 Existence result in the no-spin-hardening case . . . . . . . . . . . . . . . . . . . . . 24

5.4 Is it possible to accommodate the special case $\widehat{\sigma}_{0}=0$ in our model? . . . . . . . . . . 27

6 The limit case of vanishing characteristic length scale $L_{c} \rightarrow 0$

7 Conclusions and outlook 29

Acknowledgements $\quad 30$

\section{Introduction}

Since the celebrated work of Tresca [121], classical plasticity has been cast within the years into a beautiful framework in which both theoretical and computational aspects were examined (see e.g. $[71,76,7,114,60,118,23])$. Even perfect classical plasticity has been recently revived by $[23,38,39]$ with the use of the energetic approach for rate-independent processes developed by $[79,80]$.

On the other hand, a number of experimental results have shown size-dependencies for the material behaviour in small scales (micron/meso) (see e.g. [33, 116]). However, classical plasticity models are scale independent and therefore cannot capture those size-effects. This has led in the last thirty years to an abundant literature $([1,2,83,34,5,46,44,48,50,35,36,109])$ on theories of gradient plasticity with the aim of accommodating the experimentally observed size effects mentioned above. The so-called energetic and dissipative length scales have been involved. Moreover, effort has also been made in the past years to provide mathematical results 
for the initial boundary values problems and inequalities describing some models of gradient plasticity (see for instance, $[25,111,27,88,28,43,96,97,31]$ ). Several contributions on the computational aspects have been made as well $([26,95,15,112])$.

In most of the above-mentioned models of gradient plasticity, the plastic rotation has been ignored. If a polycrystal is treated as a randomly oriented collection of grains, it is clear that the plastic distortion $p$, which must then be seen as the average slip over all glide planes, will be nonsymmetric. Therefore, plastic spin is a reality also in polycrystalline modelling. The situation is less clear when one aims at an overall effective phenomenological description in which individual glide planes are not resolved. It is possible to show that in a purely local isotropic theory the plastic spin can be suppressed without loss of generality. The situation is again different in gradient-plasticity extensions, in which it is generally agreed that plastic spin is automatically included (e.g. [46]). However, no agreement has been reached on how to precisely include the effect of plastic spin. Our contribution aims at proposing a canonical framework to do exactly this. In $[46,11,12,107]$ models discussing the role of the plastic rotation have been proposed. For instance, [107] discusses the need to incorporate the plastic rotation in an isotropic gradient plasticity framework in order to capture some effects of a crystallographic model for a large collection of grains in a polycrystal. In the mathematical context, existence results for models with plastic spin have also been obtained ([88, 28, 31]).

The modelling challenge which we faced in the past can be explained as follows. Given the additive decomposition of the total non-symmetric distortion (the displacement gradient $\nabla u$ ), is it possible to write down a model with plastic spin (the plastic distortion $p$ is not symmetric) and allow for a defect energy depending on Nye's dislocation density tensor Curl $p$ together with an isotropic hardening response which is, however, only driven by the accumulated equivalent plastic strain $\gamma_{p}=\int_{0}^{t}\|\operatorname{sym} \dot{p}\| d s=\int_{0}^{t}\left\|\dot{\varepsilon}_{p}\right\| d s$, and cast all that in the suitable convex variational framework of the principle of maximum dissipation? In Section 3 we present exactly such a model. Our previous attempts of modelling in this direction were based on the (rate-explicit) dual flow rule but failed to satisfy the principle of maximum dissipation, [51, p. 454], see also $[53,102,20,54] .^{1}$

The new model proposed in this paper, which involves only one energetic length scale $L_{c}$ has some features which make it stand out from other proposals in rate-independent gradient plasticity with plastic spin as:

- it allows for plastic spin in a most transparent manner: for vanishing characteristic energetic length scale $L_{c} \rightarrow 0$, the plastic spin vanishes as well and the model turns into classical elasto-plasticity with symmetric plastic strain $\varepsilon_{p}=\operatorname{sym} p$ and with isotropic hardening based only on the accumulated equivalent plastic strain $\gamma_{p}=\int_{0}^{t}\|\operatorname{sym} \dot{p}\| d s=\int_{0}^{t}\left\|\dot{\varepsilon}_{p}\right\| d s$;

- it is completely isotropic and (linearized) frame-indifferent;

- it is (linearized) gauge-invariant: this means that it satisfies invariance under compatible

\footnotetext{
${ }^{1}$ It is often assumed that the plastic evolution $\dot{p}$ associated with a state of yield maximizes the dissipation relative to all admissible states. This is also equivalent to I'liushin's postulate ([72]).
} 
transformations of the reference system, i.e., in the linearized context it is invariant under

$$
\begin{array}{rlr}
\nabla u(x) & \longrightarrow \nabla u(x)+\nabla \vartheta_{p}(x) \quad \forall \vartheta_{p} \in C^{2}\left(\mathbb{R}^{3}, \mathbb{R}^{3}\right) \\
p(x) & \longrightarrow \quad p(x)+\nabla \vartheta_{p}(x) &
\end{array}
$$

which is also known as translational $\mathrm{T}(3)$-gauge invariance $([68,69,70,32])$;

- it contains only properly defined state-variables $([113,32])$. In this context, notice that, as mentioned in De Wit [24, p.1478]: "... the plastic strain [sym $p$ ] is not a state quantity, i.e., it cannot be determined from the [current] state of the body." Through a proper definition of infinitesimal state-variables, this will be clearly presented in [32].

In this model, the hardening type response is depending on a (nonlocal) kinematic term which is the non-symmetric backstress contribution $\mu L_{c}^{2}$ Curl Curl $p$, solely responsible for the appearance of plastic spin or not and related to the geometrically necessary dislocation (GND) density distribution. The isotropic hardening is related to statistically stored dislocations (SSD), which take into account a "plastically homogeneous" effect as they accumulate already during a macroscopically homogeneous deformation. Here, the SSD evolution is modelled by two isotropic hardening variables $\gamma_{p}=\int_{0}^{t}\|\operatorname{sym} \dot{p}\| d s$ and $\omega_{p}=\int_{0}^{t} \|$ skew $\dot{p} \| d s$. Hence, the full plastic distortion, and not only its symmetric part, may contribute to hardening. This is in accordance with the physical nature of plastic flow since also the evolution of the skew-symmetric part of $p$ indicates dislocation motion. It is important to emphasize that no spin cross-hardening takes place in the proposed model, i.e., the situation where plastic flow in the plastic strain $\varepsilon_{p}=\operatorname{sym} p$ causes hardening in the plastic rotation evolution of skew $p$ and vice-versa. This means that, in our model, only the accumulated equivalent plastic strain influences hardening in the evolution of the plastic strain and only the accumulated equivalent plastic rotation influences hardening in the evolution of the plastic rotation.

It is noteworthy that classical linear Prager-type kinematical hardening cannot be accommodated in the "state-variable" approach adopted here since the corresponding backstress contribution $\varepsilon_{p}=\operatorname{sym} p$ as such is not a state-variable (see e.g. [100, 113]).

Notwithstanding the use of the dislocation density tensor Curl $p$, we claim that our model is properly isotropic. In passing, notice that taking $\operatorname{Curl} \varepsilon_{p}=\operatorname{Curl} \operatorname{sym} p$ is physically inadmissible since $\operatorname{Curl} \varepsilon_{p}$ is not a defect measure for $\varepsilon_{p} \in \operatorname{Sym}(3)$. Rather, one should then take Kröner's incompatibility tensor inc $\varepsilon_{p}:=\operatorname{Curl}\left[\left(\operatorname{Curl} \varepsilon_{p}\right)^{T}\right]$. The possibilities to do exactly this will be explained in the forthcoming paper [32]. On the other hand, claims in the recent literature $[115]^{2}$ that dependencies of a model on the dislocation density tensor Curl $p$ exclude isotropy are also critically examined in [32].

It is sometimes argued that plastic spin is irrelevant in the case of isotropy $([66]){ }^{3}$ The question whether one needs a theory with plastic spin is just the question whether one can work

\footnotetext{
${ }^{2}$ Steigmann and Gupta [115, p.410] put forward that: "... the dislocation density [tensor] Curl $p$ is well-defined under symmetry transformations only if the symmetry group is discrete." From that they conclude that it is not possible to obtain an isotropic plasticity model including Curl $p$.

${ }^{3}$ Krishnan and Steigmann [66, p.722] argue that plastic spin associated with a flow rule for plastic evolution can be suppressed in the isotropic case without loss of generality. We understand that this is only true for the local theory, i.e., zero characteristic length $L_{c}=0$, as confirmed in [51, p.511].
} 
with a symmetric plastic strain tensor $\varepsilon_{p}$ as the only variable in a phenomenological plasticity theory. Our development clearly shows that claims such as in [66] are unfounded and seem to indicate that there are different notions involved of what isotropy precisely means. This subject is also discussed further in [32].

A remark concerning the mathematical treatment of single crystal plasticity is also in order. First, it is clear that such a theory is also a phenomenological model, albeit on a different scale. In the single crystal case the assumption of different glide systems lead to an immediate anisotropy of plastic flow and plastic spin is automatically included. However, the dislocation density contribution, when looked at it in detail, leads to a full gradient control of the plastic slip on each glide-plane. Therefore, the nonsymmetric plastic distortion $p$, which is the combined plastic slip on each glide plane, is automatically controlled in the standard Sobolev space $H^{1}(\Omega)$ $([110,15])$. By contrast, our isotropic framework means to give up detailed control of the plastic distortion due to additional invariance conditions that have to be respected. The effect is that there is not even an immediate $H(\mathrm{Curl})$-control of the plastic distortion. Therefore, the mathematically more challenging model is, without any doubt, the isotropic dislocation-based model with plastic spin treated here.

Notice that there are some similarities between our new isotropic model and the early one proposed by Gurtin [46]. In fact, both models share: a complete isotropic formulation, decoupled evolution equations into symmetric and skew-symmetric rates (isotropic hardening possibly coming from both), a dissipation depending also on plastic spin, the same defect and elastic energies, only an energetic length scale connected to the dislocation density tensor and both reduce to classical plasticity when the energetic length scale is zero. Now, there are also nontrivial differences between the two models. In fact, the model in [46] is visco-plastic, includes local nonsymmetric kinematical backstress due to dissipative viscoplastic hardening, it is not cast into a variational framework and does not have existence results so far. Also the model in [46] involves a novel microforce balance as well as boundary conditions on the moving elastic-plastic boundary ${ }^{4}$ and a dissipation function depending also on the gradient of the plastic distortion rate (see also [98]). The type of dissipation function considered in our model leads to an elastic region with Tresca-like branches and hence, in the flow rule in rate-explicit dual form, we get a case distinction to determine on which part of the yield surface the evolution takes place. In this, there are therefore similarities to crystal plasticity in which each glide plane has its own evolution and stresses are projected to the glide planes (see e.g. [45]). In our model the nonsymmetric Eshelby-type stress $\Sigma_{E}$ driving the plastic evolution is projected on $\mathfrak{s l}(3) \cap \operatorname{Sym}(3)$ (symmetric and traceless tensors) for the plastic strain evolution and $\mathfrak{s o}(3)$ (skew-symmetric tensors) for the plastic spin evolution.

Notice that the modelling capabilities of the model in [46] have been so far made relevant by many authors such as Bardella and co-authors [11, 13, 14, 103] and also Poh and co-authors [107]. So far, one still needs to consider a number of tests or examples to see whether the proposed model of isotropic hardening improves the results obtained in those papers or exhibits

\footnotetext{
${ }^{4}$ Unlike [46], no novel microforce balance needs to be introduced in our model. Also, in our theory, nonstandard (tangential) boundary conditions for the plastic distortion $p$ are always defined at the external boundary of the material only and the question on how to define them at a moving elastic-plastic boundary never arises. Any specific prescription of such boundary conditions at the elastic-plastic boundary could be in contradiction with the uniqueness result which we obtain for strong solutions.
} 
new features.

Let us emphasize that, while we will present the complete and rigorous mathematical existence theory to our model, the main thrust in this work is not only of analytical nature. It rather consists also in presenting that modeling framework for plastic spin which we deem to be the most suited one.

This paper is now structured as follows. In Section 2, we present some notations and definitions. In Section 3, we introduce various aspects of the model, in particular, the flow rule in both primal and dual formulations with the key role played by the dissipation function. In Sections 4 and 5, we study mathematical aspects (existence and uniqueness) of the model while in Section 6, we recover the classical plasticity framework when the characteristic length scale is set to be zero $\left(L_{c} \rightarrow 0\right)$.

\section{Some notational agreements and definitions}

Let $\Omega$ be a bounded domain in $\mathbb{R}^{3}$ with Lipschitz continuous boundary $\partial \Omega$, which is occupied by the elastoplastic body in its undeformed configuration. Let $\Gamma$ be a subset of $\partial \Omega$ with nonvanishing 2-dimensional Hausdorff measure. A material point in $\Omega$ is denoted by $x$ and the time domain under consideration is the interval $[0, T]$.

For every $a, b \in \mathbb{R}^{3}$, we let $\langle a, b\rangle_{\mathbb{R}^{3}}$ denote the scalar product on $\mathbb{R}^{3}$ with associated vector norm $|a|_{\mathbb{R}^{3}}^{2}=\langle a, a\rangle_{\mathbb{R}^{3}}$. We denote by $\mathbb{R}^{3 \times 3}$ the set of real $3 \times 3$ tensors. The standard Euclidean scalar product on $\mathbb{R}^{3 \times 3}$ is given by $\langle A, B\rangle_{\mathbb{R}^{3 \times 3}}=\operatorname{tr}\left[A B^{T}\right]$, where $B^{T}$ denotes the transpose tensor of $B$. Thus, the Frobenius tensor norm is $\|A\|^{2}=\langle A, A\rangle_{\mathbb{R}^{3 \times 3}}$. In the following we omit the subscripts $\mathbb{R}^{3}$ and $\mathbb{R}^{3 \times 3}$. The identity tensor on $\mathbb{R}^{3 \times 3}$ will be denoted by $\mathbb{1}$, so that $\operatorname{tr}(A)=$ $\langle A, \mathbb{1}\rangle$. The set $\mathfrak{s o}(3):=\left\{X \in \mathbb{R}^{3 \times 3} \mid X^{T}=-X\right\}$ is the Lie-Algebra of skew-symmetric tensors. We let $\operatorname{Sym}(3):=\left\{X \in \mathbb{R}^{3 \times 3} \mid X^{T}=X\right\}$ denote the vector space of symmetric tensors and $\mathfrak{s l}(3):=\left\{X \in \mathbb{R}^{3 \times 3} \mid \operatorname{tr}(X)=0\right\}$ be the Lie-Algebra of traceless tensors. For every $X \in \mathbb{R}^{3 \times 3}$, we set $\operatorname{sym}(X)=\frac{1}{2}\left(X+X^{T}\right)$, skew $(X)=\frac{1}{2}\left(X-X^{T}\right)$ and $\operatorname{dev}(X)=X-\frac{1}{3} \operatorname{tr}(X) \mathbb{1} \in \mathfrak{s l}(3)$ for the symmetric part, the skew-symmetric part and the deviatoric part of $X$, respectively. Quantities which are constant in space will be denoted with an overbar, e.g., $\bar{A} \in \mathfrak{s o}(3)$ for the function $A: \mathbb{R}^{3} \rightarrow \mathfrak{s o}(3)$ which is constant with constant value $\bar{A}$.

The body is assumed to undergo infinitesimal deformations. Its behaviour is governed by a set of constitutive relations. Below is a list of variables and parameters used throughout the paper:

- $u$ is the displacement of the macroscopic material points;

- $p$ is the infinitesimal plastic distortion variable which is a non-symmetric second order tensor, incapable of sustaining volumetric changes; that is, $p \in \mathfrak{s l}(3)$. The tensor $p$ represents the average plastic slip; $p$ is not a state-variable, while the rate $\dot{p}$ is;

- $e=\nabla u-p$ is the infinitesimal elastic distortion which is a non-symmetric second order tensor and is a state-variable;

- $\varepsilon_{p}=\operatorname{sym} p$ is the symmetric infinitesimal plastic strain tensor, which is also trace free, $\varepsilon_{p} \in \mathfrak{s l}(3) ; \varepsilon_{p}$ is not a state-variable; the rate $\dot{\varepsilon}_{p}=\operatorname{sym} \dot{p}$ is a state-variable; 
- skew $p$ is called plastic rotation or plastic spin;

- $\varepsilon_{e}=\operatorname{sym}(\nabla u-p)$ is the symmetric infinitesimal elastic strain tensor and is a state-variable;

- $\sigma$ is the Cauchy stress tensor which is a symmetric second order tensor and is a statevariable;

- $\sigma_{0}$ and $\widehat{\sigma}_{0}$ are the initial yield stresses for plastic strain and plastic spin, respectively and both are state-variables;

- $\sigma_{\mathrm{y}}$ and $\widehat{\sigma}_{\mathrm{y}}$ are the current yield stresses for plastic strain and plastic spin, respectively and both are state-variables;

- $f$ is the body force;

- $\operatorname{Curl} p=-\operatorname{Curl} e=\alpha$ is the dislocation density tensor satisfying the so-called Bianchi identities $\operatorname{Div} \alpha=0$ and is a state-variable;

- $\gamma_{p}=\int_{0}^{t}\|\operatorname{sym} \dot{p}\| d s=\int_{0}^{t}\left\|\dot{\varepsilon}_{p}\right\| d s$ is the accumulated equivalent plastic strain and is a state-variable;

- $\omega_{p}=\int_{0}^{t} \|$ skew $\dot{p} \| d s$ is the accumulated equivalent plastic rotation and is a state-variable; - $\int_{0}^{t} \sqrt{\dot{\gamma}_{p}^{2}+\dot{\omega}_{p}^{2}} d s=\int_{0}^{t}\|\dot{p}\| d s$ represents the accumulated equivalent plastic distortion which
is a state-variable.

For isotropic media, the fourth order isotropic elasticity tensor $\mathbb{C}_{\text {iso }}: \operatorname{Sym}(3) \rightarrow \operatorname{Sym}(3)$ is given by

$$
\mathbb{C}_{\text {iso }} \operatorname{sym} X=2 \mu \text { dev } \operatorname{sym} X+\kappa \operatorname{tr}(X) \mathbb{1}=2 \mu \operatorname{sym} X+\lambda \operatorname{tr}(X) \mathbb{1}
$$

for any second-order tensor $X$, where $\mu$ and $\lambda$ are the Lamé moduli satisfying

$$
\mu>0 \text { and } 3 \lambda+2 \mu>0
$$

and $\kappa>0$ is the bulk modulus. These conditions suffice for pointwise positive definiteness of the elasticity tensor in the sense that there exists a constant $m_{0}>0$ such that

$$
\forall X \in \mathbb{R}^{3 \times 3}: \quad\left\langle\operatorname{sym} X, \mathbb{C}_{\text {iso }} \operatorname{sym} X\right\rangle \geq m_{0}\|\operatorname{sym} X\|^{2} .
$$

The space of square integrable functions is $L^{2}(\Omega)$, while the Sobolev spaces used in this paper are:

$$
\begin{aligned}
\mathrm{H}^{1}(\Omega)= & \left\{u \in \mathrm{L}^{2}(\Omega) \mid \operatorname{grad} u \in \mathrm{L}^{2}(\Omega)\right\}, \quad \operatorname{grad}=\nabla, \\
& \|u\|_{H^{1}(\Omega)}^{2}=\|u\|_{L^{2}(\Omega)}^{2}+\|\operatorname{grad} u\|_{L^{2}(\Omega)}^{2}, \quad \forall u \in \mathrm{H}^{1}(\Omega), \\
\mathrm{H}(\operatorname{curl} ; \Omega)= & \left\{v \in \mathrm{L}^{2}(\Omega) \mid \operatorname{curl} v \in \mathrm{L}^{2}(\Omega)\right\}, \quad \operatorname{curl}=\nabla \times, \\
& \|v\|_{\mathrm{H}(\operatorname{curl} ; \Omega)}^{2}=\|v\|_{L^{2}(\Omega)}^{2}+\|\operatorname{curl} v\|_{L^{2}(\Omega)}^{2}, \quad \forall v \in \mathrm{H}(\operatorname{curl} ; \Omega) .
\end{aligned}
$$


For every $X \in C^{1}\left(\Omega, \mathbb{R}^{3 \times 3}\right)$ with rows $X_{1}, X_{2}, X_{3}$, we use in this paper the definition of Curl $X$ in $[88,119]$ :

$$
\operatorname{Curl} X=\left(\begin{array}{c}
\operatorname{curl} X_{1} \\
\operatorname{curl} X_{2} \\
\operatorname{curl} X_{3}
\end{array}\right) \in \mathbb{R}^{3 \times 3},
$$

for which Curl $\nabla v=0$ for every $v \in C^{2}\left(\Omega, \mathbb{R}^{3}\right)$. Notice that the definition of Curl $X$ above is such that $(\operatorname{Curl} X)^{T} a=\operatorname{curl}\left(X^{T} a\right)$ for every $a \in \mathbb{R}^{3}$ and this clearly corresponds to the transpose of the Curl of a tensor as defined in $[48,51]$.

The following function spaces and norms will also be used later.

$$
\begin{aligned}
\mathrm{H}\left(\mathrm{Curl} ; \Omega, \mathbb{R}^{3 \times 3}\right) & =\left\{X \in \mathrm{L}^{2}\left(\Omega, \mathbb{R}^{3 \times 3}\right) \mid \operatorname{Curl} X \in \mathrm{L}^{2}\left(\Omega, \mathbb{R}^{3 \times 3}\right)\right\}, \\
\|X\|_{\mathrm{H}(\mathrm{Curl} ; \Omega)}^{2} & =\|X\|_{L^{2}(\Omega)}^{2}+\|\operatorname{Curl} X\|_{L^{2}(\Omega)}^{2}, \quad \forall X \in \mathrm{H}\left(\mathrm{Curl} ; \Omega, \mathbb{R}^{3 \times 3}\right), \\
\mathrm{H}(\mathrm{Curl} ; \Omega, \mathbb{E}) & =\left\{X: \Omega \rightarrow \mathbb{E} \mid X \in \mathrm{H}\left(\operatorname{Curl} ; \Omega, \mathbb{R}^{3 \times 3}\right)\right\},
\end{aligned}
$$

for $\mathbb{E}:=\mathfrak{s l}(3)$ or $\operatorname{Sym}(3) \cap \mathfrak{s l}(3)$.

We also consider the space

$$
\mathrm{H}_{0}\left(\mathrm{Curl} ; \Omega, \Gamma, \mathbb{R}^{3 \times 3}\right)
$$

as the completion in the norm in (2.6) of the space $\left\{q \in C^{\infty}\left(\Omega, \Gamma, \mathbb{R}^{3 \times 3}\right)|(q \times n)|_{\Gamma}=0\right\}$. Therefore, this space generalizes the tangential Dirichlet boundary condition

$$
\left.(q \times n)\right|_{\Gamma}=0
$$

to be satisfied by the plastic distortion $p$ or the plastic strain $\varepsilon_{p}:=\operatorname{sym} p$. The space

$$
\mathrm{H}_{0}(\mathrm{Curl} ; \Omega, \Gamma, \mathbb{E})
$$

is defined as in (2.6).

The divergence operator Div on second order tensor-valued functions is also defined row-wise as

$$
\operatorname{Div} X=\left(\begin{array}{c}
\operatorname{div} X_{1} \\
\operatorname{div} X_{2} \\
\operatorname{div} X_{3}
\end{array}\right)
$$

\section{The description of the model}

\subsection{The balance equation}

The conventional macroscopic force balance leads to the equation of equilibrium

$$
\operatorname{div} \sigma+f=0
$$

in which $\sigma$ is the infinitesimal symmetric Cauchy stress and $f$ is the body force. 


\subsection{Constitutive equations.}

The constitutive equations are obtained from a free-energy imbalance together with a flow law that characterizes plastic behaviour. Since the model under study involves plastic spin by which we mean that the plastic distortion $p$ is not symmetric, we consider directly an additive decomposition of the displacement gradient $\nabla u$ into elastic and plastic components $e$ and $p$, so that

$$
\nabla u=e+p
$$

with the nonsymmetric plastic distortion $p$ incapable of sustaining volumetric changes; that is,

$$
\operatorname{tr}(p)=\operatorname{tr}(\operatorname{sym} p)=\operatorname{tr}\left(\varepsilon_{p}\right)=0 .
$$

Here, $\varepsilon_{e}=\operatorname{sym} e=\operatorname{sym}(\nabla u-p)$ is the infinitesimal elastic strain and $\varepsilon_{p}=\operatorname{sym} p$ is the plastic strain while $\operatorname{sym} \nabla u=\left(\nabla u+\nabla u^{T}\right) / 2$ is the total strain.

We consider a free energy in the additively separated form

$$
\begin{gathered}
\Psi\left(\nabla u, p, \operatorname{Curl} p, \gamma_{p}, \omega_{p}\right):=\underbrace{+\underbrace{\Psi_{\text {iso }}\left(\gamma_{p}, \omega_{p}\right)}_{\text {defect energy }(\mathrm{GND})}}_{\begin{array}{c}
\text { elastic energy } \\
\Psi_{e}^{\text {lin }}(\operatorname{sym} e)
\end{array}}, \underbrace{\Psi_{\text {curl }}^{\text {lin }}(\operatorname{Curl} p)}_{\text {hardening energy }(\mathrm{SSD})}
\end{gathered}
$$

where

$$
\begin{aligned}
& \Psi_{e}^{\operatorname{lin}}(\operatorname{sym} e):=\frac{1}{2}\left\langle\operatorname{sym} e, \mathbb{C}_{\text {iso }} \operatorname{sym} e\right\rangle, \quad \Psi_{\text {curl }}^{\operatorname{lin}}(\operatorname{Curl} p):=\frac{1}{2} \mu L_{c}^{2}\|\operatorname{Curl} p\|^{2}, \\
& \Psi_{\text {iso }}\left(\gamma_{p}, \omega_{p}\right):=\frac{1}{2} \mu \alpha_{1}\left|\gamma_{p}\right|^{2}+\frac{1}{2} \mu \alpha_{2}\left|\omega_{p}\right|^{2} .
\end{aligned}
$$

Here, $L_{c} \geq 0$ is an energetic length scale which characterizes the contribution of the defect energy density to the system, $\alpha_{1}>0$ and $\alpha_{2} \geq 0$ are nondimensional isotropic hardening constants, $\gamma_{p}$ and $\omega_{p}$ are isotropic hardening variables. The defect energy is conceptually related to geometrically necessary dislocations (GND). It is formed by the long-ranging stress-fields of excess dislocations and may be recovered by appropriate inelastic deformation. The isotropic hardening energy is related to statistically stored dislocations (SSD). ${ }^{5}$ It is formed by the local stress-fields of all dislocations and can only be recovered in thermodynamical processes such as annealing, recrystallization or chemical reactions.

\subsubsection{The derivation of the dissipation inequality}

The local free-energy imbalance states that

$$
\dot{\Psi}-\langle\sigma, \dot{e}\rangle-\langle\sigma, \dot{p}\rangle \leq 0 \text {. }
$$

\footnotetext{
${ }^{5}$ It is an easy matter to generalize the defect-energy contribution as well as the elasticity relation to the complete anisotropic setting. However, this does not add anything to enhance understanding of the paper and hence we leave these easy generalizations aside.
} 
Now we expand the first term, substitute (3.4) and get

$$
\left\langle\mathbb{C}_{\text {iso }} \operatorname{sym} e-\sigma, \operatorname{sym} \dot{e}\right\rangle-\langle\sigma, \dot{p}\rangle+\mu L_{c}^{2}\langle\operatorname{Curl} p \text {, Curl } \dot{p}\rangle+\mu \alpha_{1} \gamma_{p} \dot{\gamma}_{p}+\mu \alpha_{2} \omega_{p} \dot{\omega}_{p} \leq 0,
$$

which, using arguments from thermodynamics gives the elastic relation

$$
\sigma=\mathbb{C}_{\text {iso }} \operatorname{sym} e=2 \mu \operatorname{sym}(\nabla u-p)+\lambda \operatorname{tr}(\nabla u-p) \mathbb{1}
$$

and the local reduced dissipation inequality

$$
-\langle\sigma, \dot{p}\rangle+\mu L_{c}^{2}\langle\operatorname{Curl} p, \operatorname{Curl} \dot{p}\rangle+\mu \alpha_{1} \gamma_{p} \dot{\gamma}_{p}+\mu \alpha_{2} \omega_{p} \dot{\omega}_{p} \leq 0 .
$$

Now we integrate (3.9) over $\Omega$ and get

$$
\begin{gathered}
0 \geq \int_{\Omega}\left[-\langle\sigma, \dot{p}\rangle+\mu L_{c}^{2}\langle\operatorname{Curl} p, \operatorname{Curl} \dot{p}\rangle+\mu \alpha_{1} \gamma_{p} \dot{\gamma}_{p}+\mu \alpha_{2} \omega_{p} \dot{\omega}_{p}\right] d x \\
=-\int_{\Omega}\left[\langle\sigma, \dot{p}\rangle+\mu L_{c}^{2}\langle\operatorname{Curl} \operatorname{Curl} p, \dot{p}\rangle+\mu \alpha_{1} \gamma_{p} \dot{\gamma}_{p}+\mu \alpha_{2} \omega_{p} \dot{\omega}_{p}\right. \\
\left.+\sum_{i=1}^{3} \operatorname{div}\left(\mu L_{c}^{2} \dot{p}^{i} \times(\operatorname{Curl} p)^{i}\right)\right] d x
\end{gathered}
$$

Using the divergence theorem we obtain

$$
\begin{aligned}
\int_{\Omega}\left[\left\langle-\sigma+\mu L_{c}^{2} \operatorname{Curl} \operatorname{Curl} p, \dot{p}\right\rangle\right. & \left.+\mu \alpha_{1} \gamma_{p} \dot{\gamma}_{p}+\mu \alpha_{2} \omega_{p} \dot{\omega}_{p}\right] d x \\
& +\sum_{i=1}^{3} \int_{\partial \Omega} \mu L_{c}^{2}\left\langle\dot{p}^{i} \times(\operatorname{Curl} p)^{i}, n\right\rangle d S \leq 0 .
\end{aligned}
$$

In order to obtain a dissipation inequality in the spirit of classical plasticity, we assume that the infinitesimal plastic distortion $p$ satisfies the so-called linearized insulation condition ${ }^{6}$

$$
\sum_{i=1}^{3} \int_{\partial \Omega} \mu L_{c}^{2}\left\langle\dot{p}^{i} \times(\operatorname{Curl} p)^{i}, n\right\rangle d S=0 .
$$

Under (3.12) and splitting the rates orthogonally in the scalar product $\langle\cdot, \cdot\rangle$,

$$
\dot{p}=\operatorname{sym} \dot{p}+\text { skew } \dot{p},
$$

we then obtain a global version of the reduced dissipation inequality ${ }^{7}$

$$
\begin{aligned}
\int_{\Omega}[\langle\sigma+ & \left.\left.\Sigma_{\text {curl }}^{\operatorname{lin}}, \dot{p}\right\rangle+g_{1} \dot{\gamma}_{p}+g_{2} \dot{\omega}_{p}\right] d x \geq 0, \\
& \Leftrightarrow \int_{\Omega}\left[\left\langle\sigma+\operatorname{sym} \Sigma_{\text {curl }}^{\operatorname{lin}}, \operatorname{sym} \dot{p}\right\rangle+\left\langle\text { skew } \Sigma_{\text {curl }}^{\text {lin }}, \text { skew } \dot{p}\right\rangle+g_{1} \dot{\gamma}_{p}+g_{2} \dot{\omega}_{p}\right] d x \geq 0,
\end{aligned}
$$

\footnotetext{
${ }^{6}$ Notice that the therminology "insulation condition" has been used by Polizzotto [108] and also in references therein.

${ }^{7}$ Gurtin [46, p.4] refers to Menzel and Steinmann [78] and writes: "... but [they] satisfy the dissipation inequality [only] globally."
} 
where

$$
\Sigma_{\text {curl }}^{\operatorname{lin}}:=-\mu L_{c}^{2} \operatorname{Curl} \operatorname{Curl} p, \quad g_{1}:=-\mu \alpha_{1} \gamma_{p}, \quad g_{2}:=-\mu \alpha_{2} \omega_{p} .
$$

For further use we define the non-symmetric Eshelby-type stress tensor driving the plastic evolution

$$
\Sigma_{E}:=\sigma+\Sigma_{\mathrm{curl}}^{\operatorname{lin}},
$$

with the non-symmetry relating only to the nonlocal term $\Sigma_{\text {curl }}$. In terms of $\Sigma_{E}$ the global reduced dissipation inequality can be expressed as

$$
\int_{\Omega}\left[\left\langle\operatorname{dev} \operatorname{sym} \Sigma_{E}, \operatorname{sym} \dot{p}\right\rangle+\left\langle\text { skew } \Sigma_{E}, \text { skew } \dot{p}\right\rangle+g_{1} \dot{\gamma}_{p}+g_{2} \dot{\omega}_{p}\right] d x \geq 0 .
$$

The split used in (3.13) is a constitutive choice in that it will suggest a suitable format on how to satisfy the inequality (3.14) in all deformation processes. In our previously proposed models (see [28]), this split has not been used.

\subsubsection{The boundary conditions on the plastic distortion}

The condition (3.12) is satisfied if we assume for instance that the boundary is a perfect conductor. This means that the tangential component of $p$ vanishes on $\partial \Omega$. In the context of dislocation dynamics these conditions express the requirement that there is no flux of the Burgers vector across a hard boundary. Gurtin [46] and also Gurtin and Needleman [47] introduce the following different types of boundary conditions for the plastic distortion

$$
\begin{aligned}
\left.(\dot{p} \times n)\right|_{\Gamma_{\text {hard }}}=0 & \text { "micro-hard" (perfect conductor) } \\
\left.\dot{p}\right|_{\Gamma_{\text {hard }}}=0 & \text { "hard-slip" } \quad \text { (in the context of crystal plasticity) } \\
\left.(\text { Curl } \dot{p} \times n)\right|_{\Gamma_{\text {hard }}}=0 & \text { "micro-free" } .
\end{aligned}
$$

We specify a sufficient condition for the micro-hard boundary condition, namely

$$
\left.(p \times n)\right|_{\Gamma_{\text {hard }}}=0
$$

and assume for simplicity only $\Gamma_{\text {hard }}=\partial \Omega=\Gamma$. Note that this boundary condition constrains the plastic slip in tangential direction only, which is what we expect to happen at the physical boundary $\Gamma_{\text {hard }}$.

\subsection{The flow rule}

\subsubsection{The flow rule in its primal formulation}

Let $D: \mathbb{R}^{2} \rightarrow \mathbb{R}$ be the function defined by

$$
D(s, t):=\sqrt{\sigma_{0}^{2} s^{2}+\widehat{\sigma}_{0}^{2} t^{2}}
$$


where $\sigma_{0}, \widehat{\sigma}_{0}>0$ are the initial yield stresses for symmetric strain $\operatorname{sym} p$ and skew-symmetric spin skew $p$, respectively. ${ }^{8}$

We consider the dissipation function $\Delta$ defined by ${ }^{9}$

$$
\Delta(q, \eta, \beta):= \begin{cases}D(\|\operatorname{sym} q\|, \| \text { skew } q \|) & \text { if }\|\operatorname{sym} q\| \leq \eta \quad \text { and } \quad \| \text { skew } q \| \leq \beta \\ \infty & \text { otherwise }\end{cases}
$$

The flow rule in its primal formulation can be derived using the principle of the minimum of the dissipation function $[53,102,20]$, stating that the rate of the internal variables is the minimizer of a functional $L$ consisting of the sum of the rate of the free energy and the dissipation function with respect to appropriate boundary conditions,

$$
L=\int_{\Omega}[\dot{\Psi}+\Delta] d x
$$

The principle of the minimum of the dissipation function is closely related to the principle of maximum dissipation. Both are not physical principles but thermodynamically consistent selection rules which turn out to be convenient if no other information is available or if existing flow rules are to be extended to a more general situation. For a detailed investigation, see [54]. A very general exposition for coupled physical processes is worked out in [58, 59]. Applications to the evolution of plastic microstructures can be found in $[55,57,56,64]$.

Employing a partial integration, the stationarity conditions of (3.22) can be compactly stated as

$$
\Sigma_{p} \in \partial \Delta\left(\dot{\Gamma}_{p}\right) \quad \text { where } \Sigma_{p}=\left(\sigma+\Sigma_{\text {curl }}^{\operatorname{lin}}, g_{1}, g_{2}\right) \quad \text { and } \quad \Gamma_{p}=\left(p, \gamma_{p}, \omega_{p}\right)
$$

and where $\partial \Delta$ denotes the subdifferential of $\Delta$. That is, for $\Sigma_{p} \in \partial \Delta\left(\dot{\Gamma}_{p}\right)$ we must have

$$
\begin{aligned}
\Delta(\bar{\Gamma}) & \geq \Delta\left(\dot{\Gamma}_{p}\right)+\left\langle\Sigma_{p}, \bar{\Gamma}-\dot{\Gamma}_{p}\right\rangle \\
& =\Delta\left(\dot{\Gamma}_{p}\right)+\left\langle\sigma+\Sigma_{\text {curl }}, q-\dot{p}\right\rangle+g_{1}\left(\eta-\dot{\gamma}_{p}\right)+g_{2}\left(\beta-\dot{\omega}_{p}\right) \\
& =\Delta\left(\dot{\Gamma}_{p}\right)+\left\langle\Sigma_{E}, q-\dot{p}\right\rangle+g_{1}\left(\eta-\dot{\gamma}_{p}\right)+g_{2}\left(\beta-\dot{\omega}_{p}\right),
\end{aligned}
$$

for every $\bar{\Gamma}=(q, \eta, \beta)$. By choosing $\bar{\Gamma}=(0,0,0)$ in (3.24), we get the reduced dissipation inequality in pointwise form

$$
\begin{aligned}
\left\langle\Sigma_{E}, \dot{p}\right\rangle & +g_{1} \dot{\gamma}_{p}+g_{2} \dot{\omega}_{p} \geq 0, \\
& \left.\Leftrightarrow\left\langle\operatorname{dev} \operatorname{sym} \Sigma_{E}, \operatorname{sym} \dot{p}\right\rangle+\left\langle\text { skew } \Sigma_{E}, \text { skew } \dot{p}\right\rangle+g_{1} \dot{\gamma}_{p}+g_{2} \dot{\omega}_{p}\right] \geq 0
\end{aligned}
$$

\footnotetext{
${ }^{8}$ Both values together will define the elastic domain in the stress space and this domain must have nonempty interior. Therefore, we need $\sigma_{0}, \widehat{\sigma}_{0}>0$. Without isotropic hardening the elastic domain turns out to be $\left\{\Sigma_{E} \in \mathbb{R}^{3 \times 3} \mid\left\|\operatorname{dev} \operatorname{sym} \Sigma_{E}\right\| \leq \sigma_{0}, \|\right.$ skew $\left.\Sigma_{E} \| \leq \widehat{\sigma}_{0}\right\}$.

${ }^{9}$ Gurtin [46, p.2554] notes: "One would expect that, plastically, the material response to spin differs to straining, and that straining and spin each incur dissipation." Gurtin's choice of the dissipation function in [46] corresponds to $\widehat{\sigma}_{0}=\chi \sigma_{0} \geq 0$ in (3.20). Also, Gurtin [46, p.2558] takes $\chi \rightarrow 0$ formally and recovers classical plasticity. If we want to take $\widehat{\sigma}_{0} \rightarrow 0$ in our setting, then we encounter a problem described in Section 5.4.
} 


\subsubsection{The flow rule in its dual formulation}

While the flow rule in the primal formulation is extremely condensed and will allow us a mathematical treatment (existence), we need the representation of the flow rule in the dual formulation in most computational implementations and for the uniqueness proof in Section 4.4. For this formulation of the flow rule we need to derive the set of admissible (generalized) stresses $\mathcal{E}$ (the elastic domain) corresponding to the dissipation function $\Delta$. According to the principle of maximum dissipation, ${ }^{10}$ the flow rule in dual form is formulated in the context of convex analysis as

$$
\dot{\Gamma}_{p} \in N_{\mathcal{E}}\left(\Sigma_{p}\right) \quad \Leftrightarrow \quad\left\langle\dot{\Gamma}_{p}, \bar{\Sigma}-\Sigma_{p}\right\rangle \leq 0 \quad \forall \bar{\Sigma} \in \mathcal{E},
$$

where $N_{\mathcal{E}}\left(\Sigma_{p}\right)$ is the normal cone to the set $\mathcal{E}$ of admissible stresses at $\Sigma_{p}$. Therefore, we need to find the set $\mathcal{E}$. In the context of convex analysis, the indicator function $I_{\mathcal{E}}$ of the set $\mathcal{E}$ is the Fenchel-Legendre conjugate of the dissipation function $\Delta$. Let us find the set $\mathcal{E}$ whose interior $\operatorname{int}(\mathcal{E})$ is the elastic domain and its boundary $\partial \mathcal{E}$ is the yield surface.

For $\Sigma_{p}=\left(\Sigma_{E}, g_{1}, g_{2}\right)$ with $\Sigma_{E}:=\sigma+\Sigma_{\text {curl }}$, we have

$$
\begin{aligned}
I_{\mathcal{E}}\left(\Sigma_{p}\right) & =\sup \left\{\left\langle\Sigma_{p}, \Gamma\right\rangle-\Delta(\Gamma) \mid \Gamma=(q, \eta, \beta)\right\} \\
& =\sup \left\{\left\langle\Sigma_{E}, q\right\rangle+g_{1} \eta+g_{2} \beta-\Delta(q, \eta, \beta) \mid\|\operatorname{sym} q\| \leq \eta, \| \text { skew } q \| \leq \beta\right\} \\
& =\sup _{q}\left[\sup _{\eta, \beta}\left\{\left\langle\Sigma_{E}, q\right\rangle+g_{1} \eta+g_{2} \beta-\Delta(q, \eta, \beta) \mid\|\operatorname{sym} q\| \leq \eta, \| \text { skew } q \| \leq \beta\right\}\right] \\
& =\sup _{q}\left\{\left\langle\Sigma_{E}, q\right\rangle+g_{1}\|\operatorname{sym} q\|+g_{2} \| \text { skew } q \|-\Delta(q,\|\operatorname{sym} q\|, \| \text { skew } q \|)\right\} \\
& =\sup _{q}\left\{\begin{array}{c}
\left\langle\operatorname{dev} \operatorname{sym} \Sigma_{E}, \operatorname{sym} q\right\rangle+\left\langle\text { skew } \Sigma_{E}, \text { skew } q\right\rangle \\
\left.\quad+g_{1}\|\operatorname{sym} q\|+g_{2} \| \text { skew } q \|-\Delta(q,\|\operatorname{sym} q\|, \| \text { skew } q \|)\right\},
\end{array}\right.
\end{aligned}
$$

where the supremum with respect to $\eta$ and $\beta$ is achieved for $\eta=\|\operatorname{sym} q\|$ and $\beta=\|$ skew $q \|$ since $g_{1} \leq 0$ and $g_{2} \leq 0$.

Now taking the supremum with respect to $q$ and using the fact that $\left\langle\Sigma_{E}, q\right\rangle$ is maximum with respect to $q$ only when $q$ is in the direction of $\Sigma_{E}$, we find that it is not restrictive to assume that

$$
\operatorname{sym} q=s \frac{\operatorname{dev} \operatorname{sym} \Sigma_{E}}{\left\|\operatorname{dev} \operatorname{sym} \Sigma_{E}\right\|} \quad \text { and } \quad \text { skew } q=t \frac{\text { skew } \Sigma_{E}}{\| \text { skew } \Sigma_{E} \|} .
$$

We then obtain

$$
I_{\mathcal{E}}\left(\Sigma_{p}\right)=\sup _{s \geq 0, t \geq 0}\left\{s\left(\left\|\operatorname{dev} \operatorname{sym} \Sigma_{E}\right\|+g_{1}\right)+t\left(\| \text { skew } \Sigma_{E} \|+g_{2}\right)-\sqrt{\sigma_{0}^{2} s^{2}+\widehat{\sigma}_{0}^{2} t^{2}}\right\} .
$$

To simplify the function of $s$ and $t$ to be maximized in (3.29), we set

$$
A:=\left\|\operatorname{dev} \operatorname{sym} \Sigma_{E}\right\|+g_{1} \quad \text { and } \quad B:=\| \text { skew } \Sigma_{E} \|+g_{2},
$$

and hence,

$$
I_{\mathcal{E}}\left(\Sigma_{p}\right)=\sup _{s \geq 0, t \geq 0}\left\{A s+B t-\sqrt{\sigma_{0}^{2} s^{2}+\widehat{\sigma}_{0}^{2} t^{2}}\right\}
$$

\footnotetext{
${ }^{10}$ which, again, is not a principle, but a useful and often made simplifying assumption.
} 
Notice immediately that

$$
\begin{aligned}
I_{\mathcal{E}}\left(\Sigma_{p}\right)= & \sup _{s \geq 0, t \geq 0}\left\{\frac{A}{\sigma_{0}} s+\frac{B}{\widehat{\sigma}_{0}} t-\sqrt{s^{2}+t^{2}}\right\} \\
= & \text { if } \begin{cases}A \leq \sigma_{0} & \text { if } \quad B \leq 0 \\
B \leq \widehat{\sigma}_{0} & \text { if } \quad A \leq 0 \\
\frac{A^{2}}{\sigma_{0}^{2}}+\frac{B^{2}}{\widehat{\sigma}_{0}^{2}} \leq 1 & \text { if }\left\{\begin{array}{l}
A \geq 0 \\
B \geq 0
\end{array}\right.\end{cases}
\end{aligned}
$$

Let us now introduce a set $\mathcal{K} \subset \mathbb{R}^{2}$ needed for elucidating the branching behaviour of our flow rule and defined by

$$
\mathcal{K}:=\mathcal{K}_{1} \cup \mathcal{K}_{2} \cup \mathcal{K}_{3}
$$

where

$$
\begin{aligned}
& \mathcal{K}_{1}=\left(-\infty, \sigma_{0}\right] \times(-\infty, 0], \quad \mathcal{K}_{2}=(-\infty, 0] \times\left(-\infty, \widehat{\sigma}_{0}\right], \\
& \mathcal{K}_{3}=\left\{(A, B) \in \mathbb{R}^{+} \times \mathbb{R}^{+} \mid \frac{A^{2}}{\sigma_{0}^{2}}+\frac{B^{2}}{\widehat{\sigma}_{0}^{2}} \leq 1\right\} .
\end{aligned}
$$

The set $\mathcal{K}$ in the $A B$-plane is represented graphically in Figure 1 . Notice that the set $\mathcal{K}$ itself is not the elastic domain. In our setting, the elastic domain is then defined as the interior of the set

$$
\mathcal{E}=\left\{\left(\Sigma_{E}, g_{1}, g_{2}\right) \in \mathbb{R}^{3 \times 3} \times \mathbb{R}^{-} \times \mathbb{R}^{-} \mid\left(\| \text {dev sym } \Sigma_{E}\left\|+g_{1},\right\| \text { skew } \Sigma_{E} \|+g_{2}\right) \in \mathcal{K}\right\} .
$$

In other terms, the set $\mathcal{E}$, which is also called the set of admissible stresses, is expressed as

$$
\mathcal{E}=\mathcal{E}_{1} \cup \mathcal{E}_{2} \cup \mathcal{E}_{3}
$$

where

$$
\begin{aligned}
& \mathcal{E}_{1}=\left\{\left(\Sigma_{E}, g_{1}, g_{2}\right) \mid \| \text { dev sym } \Sigma_{E}\left\|\leq-g_{1}+\sigma_{0}, \quad\right\| \text { skew } \Sigma_{E} \| \leq-g_{2} \quad\right\}, \\
& \mathcal{E}_{2}=\left\{\left(\Sigma_{E}, g_{1}, g_{2}\right) \mid \| \text { dev sym } \Sigma_{E}\left\|\leq-g_{1}, \quad\right\| \text { skew } \Sigma_{E} \| \leq-g_{2}+\widehat{\sigma}_{0}\right\}, \\
& \mathcal{E}_{3}=\left\{\begin{array}{ccc}
\left(\Sigma_{E}, g_{1}, g_{2}\right) \mid & \| \text { dev sym } \Sigma_{E} \| \geq-g_{1}, & \| \text { skew } \Sigma_{E} \| \geq-g_{2}, \\
\text { and } & \frac{\left(\| \text { dev sym } \Sigma_{E} \|+g_{1}\right)^{2}}{\sigma_{0}^{2}}+\frac{\left(\| \text { skew } \Sigma_{E} \|+g_{2}\right)^{2}}{\widehat{\sigma}_{0}^{2}} \leq 1
\end{array}\right\} .
\end{aligned}
$$

Hence, the yield surface is given by

$$
\partial \mathcal{E}=\mathcal{S}_{1} \cup \mathcal{S}_{2} \cup \mathcal{S}_{3}
$$




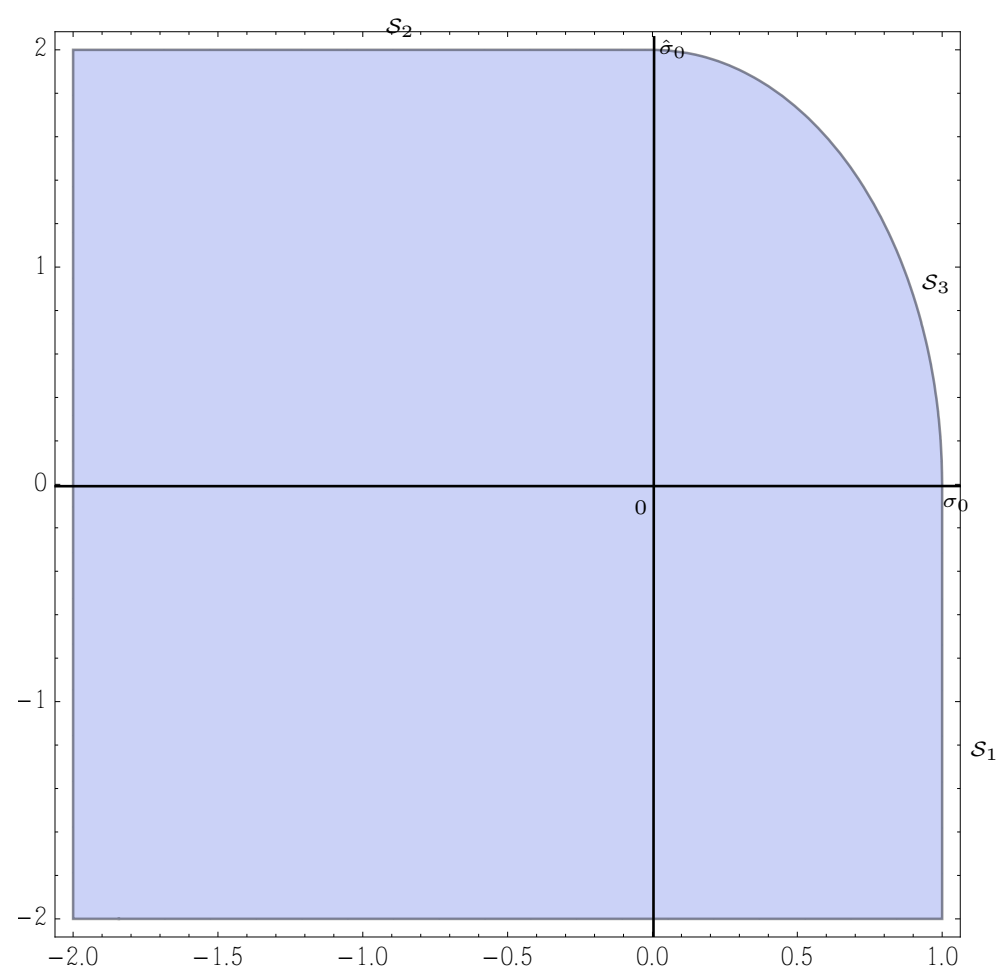

Figure 1: The set $\mathcal{K}$ in the $A B$-plane. On $\mathcal{S}_{1}$ the flow is only driven by the symmetric rate part sym $\dot{p}$ as skew $\dot{p}=0$. On $\mathcal{S}_{2}$ the flow is only driven by the skew-symmetric rate part skew $\dot{p}$ as $\operatorname{sym} \dot{p}=0$. On $\mathcal{S}_{3}$ the flow is driven by both symmetric and skew-symmetric rate parts skew $\dot{p}$ and $\operatorname{sym} \dot{p}$.

with

$$
\begin{aligned}
& \mathcal{S}_{1}=\left\{\left(\Sigma_{E}, g_{1}, g_{2}\right) \mid \| \text { dev sym } \Sigma_{E}\left\|=-g_{1}+\sigma_{0}, \quad\right\| \text { skew } \Sigma_{E} \| \leq-g_{2} \quad\right\}, \\
& \mathcal{S}_{2}=\left\{\left(\Sigma_{E}, g_{1}, g_{2}\right) \mid \| \text { dev sym } \Sigma_{E}\left\|\leq-g_{1}, \quad\right\| \text { skew } \Sigma_{E} \|=-g_{2}+\widehat{\sigma}_{0}\right\},
\end{aligned}
$$

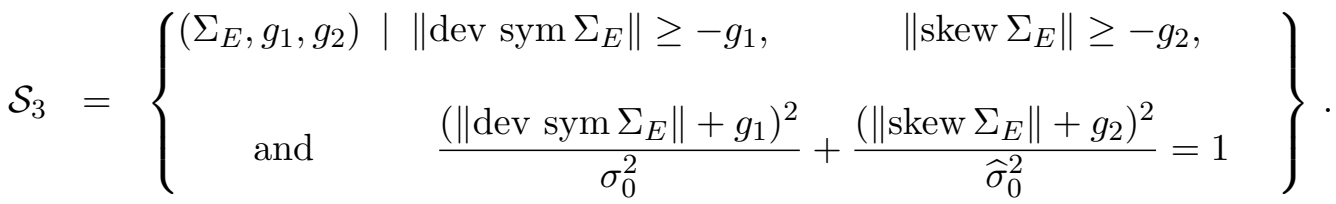

Remark 3.1 We could consider a more general dissipation function corresponding e.g. to the function

$$
\widehat{D}(s, t):=r_{1} s+r_{2} t+\sqrt{\sigma_{0}^{2} s^{2}+\widehat{\sigma}_{0}^{2} t^{2}} \quad \text { with } r_{1}, r_{2} \geq 0 .
$$

For such a choice, we get by easy calculations, the set of admissible stresses

$$
\overline{\mathcal{E}}=\left\{\left(\Sigma_{E}, g_{1}, g_{2}\right) \in \mathbb{R}^{3 \times 3} \times \mathbb{R}^{-} \times \mathbb{R}^{-} \mid\left(\| \text {dev sym } \Sigma_{E}\left\|+g_{1}+r_{1},\right\| \text { skew } \Sigma_{E} \|+g_{2}+r_{2}\right) \in \mathcal{K}\right\},
$$


which corresponds to a dilation of the set in (3.34). Such a choice will not add any particular feature to the current model. In fact, this simply corresponds to the expansion of the initial elastic domain (i.e. before isotropic hardening takes place).

Let us briefly discuss the evolution of the yield surface. The hardening behavior will depend on the values of the moduli $\alpha_{1}, \alpha_{2}$, and on the location of the generalized stress state $\left(\Sigma_{E}, g_{1}, g_{2}\right)$ on the yield surface. There are four different possibilities, displayed in Fig. 2. For clarity, we summarize the various cases in Table 1.

\begin{tabular}{|c|cccc|}
\hline & $\alpha_{1}=0, \alpha_{2}=0$ & $\alpha_{1}>0, \alpha_{2}=0$ & $\alpha_{1}=0, \alpha_{2}>0$ & $\alpha_{1}>0, \alpha_{2}>0$ \\
\hline$\left(\Sigma_{E}, g_{1}, g_{2}\right) \in \mathcal{S}_{1}$ & a. $)$ & b. $)$ & a. $)$ & b. $)$ \\
$\left(\Sigma_{E}, g_{1}, g_{2}\right) \in \mathcal{S}_{2}$ & a.) & a. $)$ & c.) & c.) \\
$\left(\Sigma_{E}, g_{1}, g_{2}\right) \in \mathcal{S}_{3}$ & a. $)$ & b. $)$ & c. $)$ & d.) \\
\hline
\end{tabular}

Table 1: Evolution of the yield surface, different hardening scenarios.

Next, our goal is to present a strong and a weak formulation of the model, followed by two existence results for which there is an important distinction between the cases $\alpha_{2}>0$ and $\alpha_{2}=0$ in the free-energy $\Psi$ in (3.4)-(3.5).

\section{The complete mathematical formulation in the case $\alpha_{2}>0$}

In this section, we present the full description of the model in the case $\alpha_{2}>0$ in the free-energy $\Psi$ in (3.4)-(3.5) as well as a corresponding existence result. The case $\alpha_{2}>0$ means that there is always isotropic hardening in the spin-evolution equation. We recall that the dissipation function $\Delta$ is given in (3.21) and the yield function in the case $\sigma_{0}>0$ and $\widehat{\sigma}_{0}>0$ (see (3.37)) is given by

$$
\phi\left(\Sigma_{p}\right):= \begin{cases}\| \text { dev sym } \Sigma_{E} \|+g_{1}-\sigma_{0} & \text { on } \mathcal{S}_{1} \\ \| \text { skew } \Sigma_{E} \|+g_{2}-\widehat{\sigma}_{0} & \text { on } \mathcal{S}_{2} \\ \frac{\left(\| \text { dev sym } \Sigma_{E} \|+g_{1}\right)^{2}}{\sigma_{0}^{2}}+\frac{\left(\| \text { skew } \Sigma_{E} \|+g_{2}\right)^{2}}{\widehat{\sigma}_{0}^{2}}-1 & \text { on } \mathcal{S}_{3} .\end{cases}
$$

\subsection{The strong formulation}

The strong formulation of the model consists in finding:

(i) the displacement $u \in \mathrm{H}^{1}\left(0, T ; \mathrm{H}_{0}^{1}\left(\Omega, \Gamma, \mathbb{R}^{3}\right)\right)$,

(ii) the infinitesimal plastic distortion $p \in \mathrm{H}^{1}\left(0, T ; \mathrm{L}^{2}(\Omega, \mathfrak{s l}(3))\right)$ with

$$
\operatorname{Curl} p \in \mathrm{H}^{1}\left(0, T ; \mathrm{L}^{2}\left(\Omega, \mathbb{R}^{3 \times 3}\right)\right) \quad \text { and } \quad \text { Curl Curl } p \in \mathrm{H}^{1}\left(0, T ; \mathrm{L}^{2}\left(\Omega, \mathbb{R}^{3 \times 3}\right)\right) \text {, }
$$




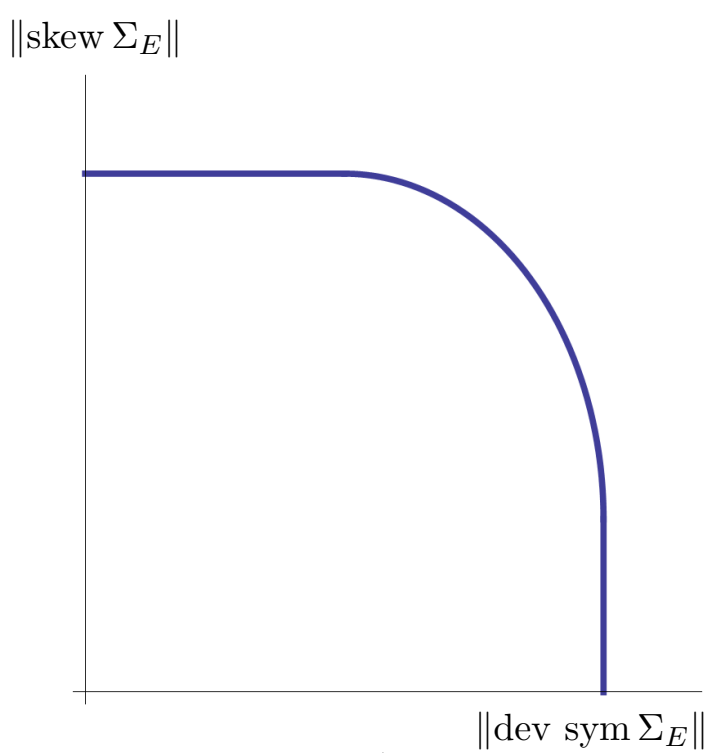

a.)

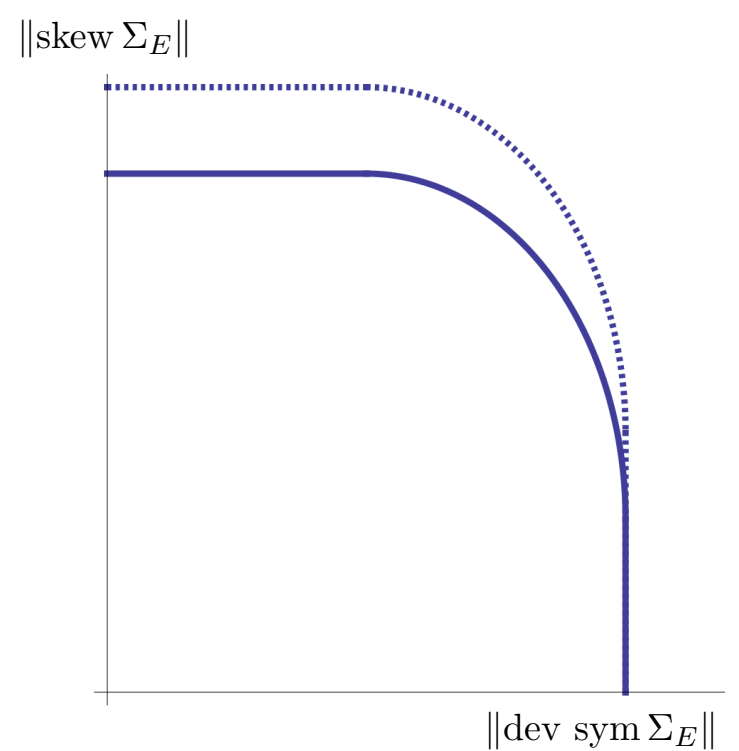

c.)

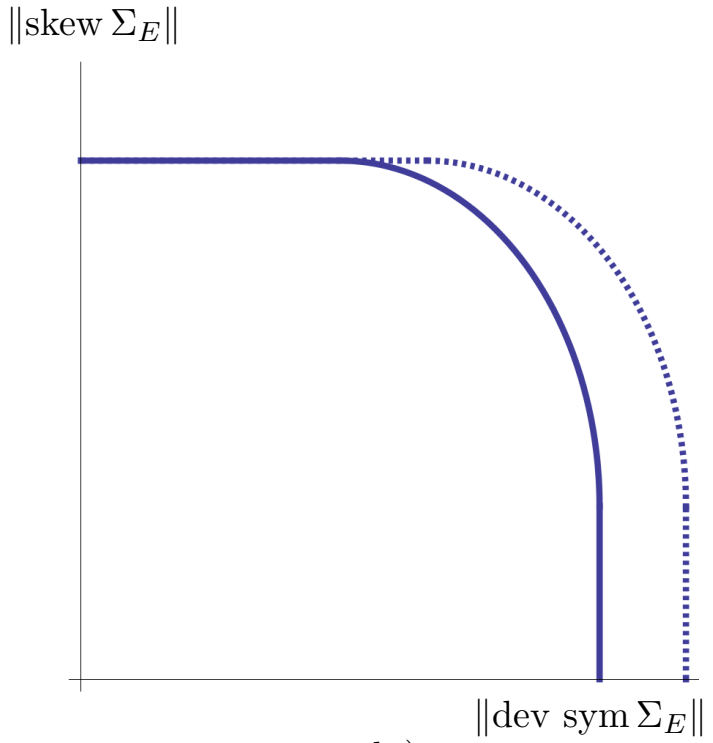

b.)

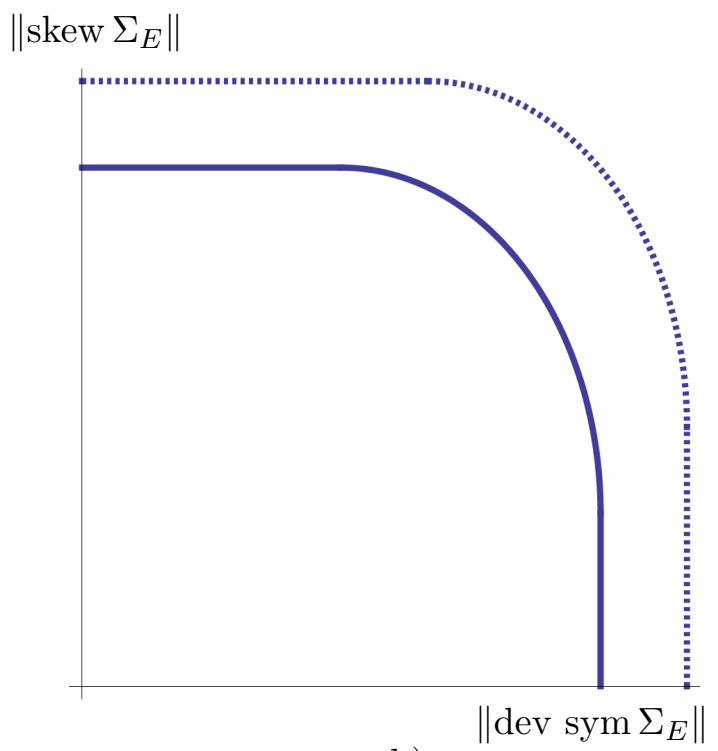

d.)

Figure 2: Evolution of the yield surface by isotropic hardening: original yield surface depicted by solid line, evolved yield surface by dashed line. Different cases: a.) no evolution of yield surface, b.) expansion in direction of $\left\|\operatorname{dev} \operatorname{sym} \Sigma_{E}\right\|$, c.) expansion in direction of $\|$ skew $\Sigma_{E} \|$, d.) expansion in both directions. 
(iii) the internal isotropic hardening variables $\gamma_{p}, \omega_{p} \in \mathrm{H}^{1}\left(0, T ; \mathrm{L}^{2}(\Omega)\right)$

such that the content of Table 2 holds.

\subsection{The weak formulation}

Assume that the problem in Section 4.1 has a solution $w=\left(u, \Gamma_{p}\right)$ with $\Gamma_{p}=\left(p, \gamma_{p}, \omega_{p}\right)$. Let $v \in H^{1}\left(\Omega, \mathbb{R}^{3}\right)$ with $v_{\mid \Gamma}=0$. Multiply the equilibrium equation with $v-\dot{u}$ and integrate in space by parts and use the symmetry of $\sigma$ and the elasticity relation to get

$$
\int_{\Omega}\left\langle\mathbb{C}_{\text {iso }} \operatorname{sym}(\nabla u-p), \operatorname{sym}(\nabla v-\nabla \dot{u})\right\rangle d x=\int_{\Omega} f(v-\dot{u}) d x .
$$

Now, for any $q \in C^{\infty}(\bar{\Omega}, \mathfrak{s l}(3))$ such that $q \times n=0$ on $\Gamma$ and any $\eta, \beta \in L^{2}(\Omega)$, we integrate (3.24) over $\Omega$, integrate by parts the term with Curl Curl using the boundary conditions

$$
(q-\dot{p}) \times n=0 \text { on } \Gamma, \quad \operatorname{Curl} p \times n=0 \text { on } \partial \Omega \backslash \Gamma
$$

and get for $\bar{\Gamma}=(q, \eta, \beta)$

$$
\begin{aligned}
\int_{\Omega} \Delta(\bar{\Gamma}) d x-\int_{\Omega} \Delta\left(\dot{\Gamma}_{p}\right) d x-\int_{\Omega}\left\langle\mathbb{C}_{\text {iso }}(\operatorname{sym} \nabla u-\operatorname{sym} p), \operatorname{sym} q-\operatorname{sym} \dot{p}\right\rangle d x \\
\quad+\mu L_{c}^{2} \int_{\Omega}\left[\langle\operatorname{Curl} p, \operatorname{Curl}(q-\dot{p})\rangle+\mu \alpha_{1} \gamma_{p}\left(\eta-\dot{\gamma}_{p}\right)+\mu \alpha_{2} \omega_{p}\left(\beta-\dot{\omega}_{p}\right)\right] d x \geq 0 .
\end{aligned}
$$

Now adding up (4.2) and (4.3) we get the following weak formulation of the problem in Section 4.1 in the form of a variational inequality:

$$
\begin{aligned}
\int_{\Omega}\left\langle\mathbb{C}_{\text {iso }}(\operatorname{sym} \nabla u-\operatorname{sym} p),(\operatorname{sym} \nabla v-\operatorname{sym} q)-(\operatorname{sym} \nabla \dot{u}-\operatorname{sym} \dot{p})\right\rangle d x \\
+\mu L_{c}^{2} \int_{\Omega}\langle\operatorname{Curl} p, \operatorname{Curl}(q-\dot{p})\rangle+\int_{\Omega}\left[\mu \alpha_{1} \gamma_{p}\left(\eta-\dot{\gamma}_{p}\right)+\mu \alpha_{2} \omega_{p}\left(\beta-\dot{\omega}_{p}\right)\right] d x \\
\quad+\int_{\Omega} \Delta(\bar{\Gamma}) d x-\int_{\Omega} \Delta\left(\dot{\Gamma}_{p}\right) d x \geq \int_{\Omega} f(v-\dot{u}) d x .
\end{aligned}
$$

\subsection{Existence result for the weak formulation}

To prove the existence result for the weak formulation (4.4), we closely follow the abstract machinery developed by Han and Reddy in [60] for mathematical problems in geometrically linear classical plasticity and used for instance in $[25,111,88,28,31]$ for models of gradient plasticity. Precisely, we will need the following theorem.

Theorem 4.1 ([60, Theorem 6.19])

Let $\mathrm{Z}$ be a Hilbert space and let $\mathrm{W}$ be a nonempty closed convex cone of $\mathrm{Z}$. Consider the following problem: find $w \in \mathrm{H}^{1}([0, T] ; Z)$ with $w(0)=0$ such that for almost every $t \in[0, T], \dot{w}(t) \in \mathrm{W}$ and

$$
\boldsymbol{a}(w, z-\dot{w})+j(z)-j(\dot{w}) \geq\langle\ell, z-\dot{w}\rangle \text { for every } z \in \mathbf{W} .
$$

Assume that the following hold: 


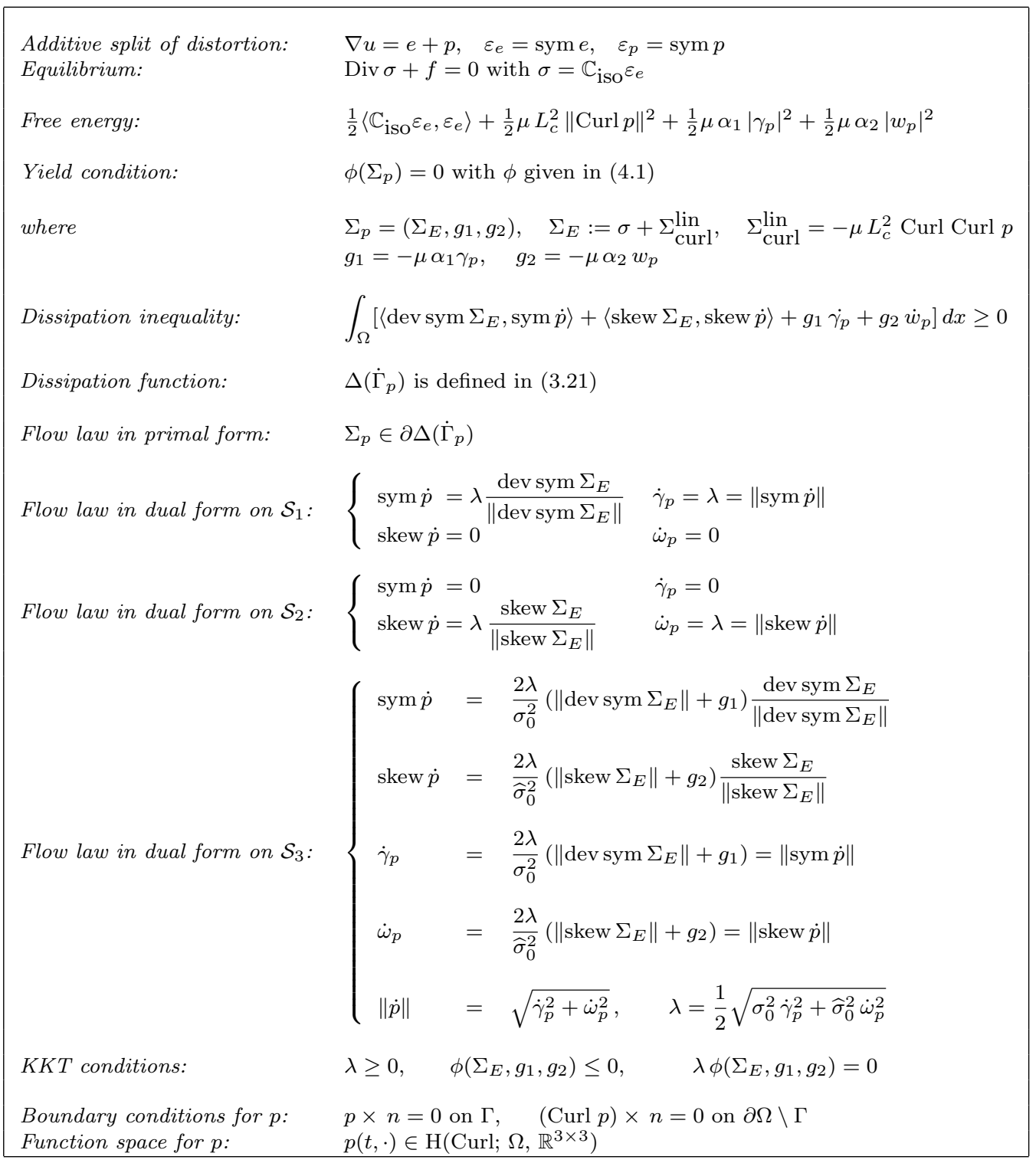

Table 2: The model with isotropic hardening and plastic spin: the case $\alpha_{2}>0$. Because of the control of the $L^{2}$-norm of both isotropic hardening variables $\eta$ and $\beta$ and the constraints $\|\operatorname{sym} q\| \leq \eta$ and $\|$ skew $q \| \leq \beta$, the function space for the non-symmetric plastic distortion $p$ is easily seen to be $\mathrm{H}\left(\mathrm{Curl} ; \Omega, \mathbb{R}^{3 \times 3}\right)$. Note that the dual formulation of the flow rule needs a case distinction depending on the branches $\mathcal{S}_{i}$ of the yield surface while the primal formulation does not need it. Clearly, isotropic hardening for the plastic strain depends only on the accumulated equivalent plastic strain and isotropic hardening for the plastic rotation depends only on the accumulated equivalent plastic rotation. Therefore, there is no spin cross-hardening. In this flow rule in dual form we appreciate a Tresca like behaviour (see [61]) in that we have to use a case distinction to determine on which part of the yield surface the evolution takes place.

1. the bilinear form $\boldsymbol{a}$ is symmetric, continuous on $\mathrm{Z}$ and coercive on $\mathrm{W}$, i.e., there exist 


$$
\begin{aligned}
& C>0 \text { and } \alpha>0 \text { such that } \\
& \quad \boldsymbol{a}(w, z) \leq C\|w\|_{Z}\|z\|_{Z} \quad \forall w, z \in \mathrm{Z} \quad \text { and } \quad \boldsymbol{a}(z, z) \geq \alpha\|z\|_{Z}^{2} \quad \forall z \in \mathrm{W} ;
\end{aligned}
$$

2. $\ell \in \mathrm{H}^{1}\left([0, T] ; \mathrm{Z}^{\prime}\right)$ with $\ell(0)=0$.

3. the functional $j$ is non-negative, convex, lower continuous and positively 1-homogeneous W, i.e., $j(s z)=|s| j(z) \quad \forall s \in \mathbb{R}, \quad \forall z \in \mathbf{Z}$.

Then the problem (4.5) has a solution $w \in \mathrm{H}^{1}([0, T] ; \mathrm{Z})$.

Remark 4.1 Theorem 4.1 above corresponds to models where the body is initially unlodaded that is, $\ell(0)=0$ and initially undeformed that is, $w(0)=0$. In the case of models with plastically pre-deformed bodies, Theorem 4.1 is still valid with the initial condition $w(0)=w_{0}$ where $w_{0} \in W$ is the solution of the elliptic variational inequality

$$
\boldsymbol{a}\left(w_{0}, z-w_{0}\right)+j(z)-j\left(w_{0}\right) \geq\left\langle\ell(0), z-w_{0}\right\rangle \text { for every } z \in \mathbf{W} .
$$

Now, in order to get an existence result for the weak formulation (4.4) through the abstract result in Theorem 4.1, we write (4.4) as (4.5) with

$$
\begin{aligned}
\boldsymbol{a}(w, z)= & \int_{\Omega}\left[\left\langle\mathbb{C}_{\text {iso }}(\operatorname{sym} \nabla u-\operatorname{sym} p), \operatorname{sym} \nabla v-\operatorname{sym} q\right\rangle+\mu L_{c}^{2}\langle\operatorname{Curl} p, \operatorname{Curl} q\rangle\right. \\
& \left.+\mu \alpha_{1} \gamma_{p} \eta+\mu \alpha_{2} \omega_{p} \beta\right] d x, \\
j(z)= & \int_{\Omega} \Delta(q, \eta, \beta) d x, \\
\langle\ell, z\rangle= & \int_{\Omega} f v d x,
\end{aligned}
$$

for $w=\left(u, p, \gamma_{p}, \omega_{p}\right)$ and $z=(v, q, \eta, \beta)$ in $\mathbf{Z}$.

The Hilbert space $\mathbf{Z}$ and the closed convex subset $\mathrm{W}$ will be constructed later in such a way that the functionals $\boldsymbol{a}, j$ and $\ell$ satisfy the assumptions of Theorem 4.1.

We let

$$
\begin{aligned}
\mathrm{V} & =\mathrm{H}_{0}^{1}\left(\Omega, \Gamma_{\mathrm{D}}, \mathbb{R}^{3}\right)=\left\{v \in \mathrm{H}^{1}\left(\Omega, \mathbb{R}^{3}\right) \mid v_{\mid \Gamma}=0\right\}, \\
\mathrm{Q} & =\mathrm{H}_{0}(\operatorname{Curl} ; \Omega, \Gamma, \mathfrak{s l}(3)), \\
\Lambda & =L^{2}(\Omega), \\
\mathrm{Z} & =\mathrm{V} \times \mathrm{Q} \times \Lambda^{2}, \\
\mathrm{~W} & =\{z=(v, q, \eta, \beta) \in \mathrm{Z} \mid \| \text { sym } q \| \leq \eta \text { and } \| \text { skew } q \| \leq \beta\},
\end{aligned}
$$

and define the norms

$$
\begin{aligned}
\|v\|_{V} & :=\|\nabla v\|_{L^{2}}, \quad\|q\|_{Q}:=\|q\|_{\mathrm{H}(\mathrm{Curl} ; \Omega)}, \\
\|z\|_{Z}^{2} & :=\|v\|_{V}^{2}+\|q\|_{Q}^{2}+\|\eta\|_{L^{2}}^{2}+\|\beta\|_{L^{2}}^{2} \quad \text { for } z=(v, q, \eta, \beta) \in \mathbf{Z} .
\end{aligned}
$$


We will assume that the body is initially unloaded and undeformed and this corresponds to assuming that $f(x, 0)=0$ for almost all $x \in \Omega$ with homogeneous initial conditions. We then get the following existence result for the weak formulation (4.4).

Theorem 4.2 Under the choices of the Hilbert space $\mathrm{Z}$ and the closed convex cone $\mathrm{W}$ in (4.10)(4.11) with the norms in (4.12) and the functionals $\boldsymbol{a}, j$ and $\ell$ in (4.8)-(4.9), the weak formulation (4.4) when written as the variational inequality of the second kind (4.5) has a solution $w=\left(u, p, \gamma_{p}, \omega_{p}\right) \in \mathrm{H}^{1}([0, T] ; \mathrm{Z})$ with $\dot{w} \in \mathrm{L}^{2}([0, T] ; \mathrm{W})$.

Proof: The functionals $j$ and $\ell$ trivially satisfy the asumptions of Theorem 4.1. So, the key issue here is the coercivity of the bilinear form $\boldsymbol{a}$ on the set $\mathbf{W}$. Let therefore $z=(v, q, \eta, \beta) \in \mathbf{W}$.

$$
\begin{gathered}
\boldsymbol{a}(z, z) \geq m_{0}\|\operatorname{sym} \nabla v-\operatorname{sym} q\|_{2}^{2}(\text { from }(2.3)) \\
\quad+\mu L_{c}^{2}\|\operatorname{Curl} q\|_{2}^{2}+\mu \alpha_{1}\|\eta\|_{2}^{2}+\mu \alpha_{2}\|\beta\|_{2}^{2} \\
=m_{0}\left[\|\operatorname{sym} \nabla v\|_{2}^{2}+\|\operatorname{sym} q\|_{2}^{2}-2\langle\operatorname{sym} \nabla v, \operatorname{sym} p\rangle\right] \\
\quad+\mu L_{c}^{2}\|\operatorname{Curl} q\|_{2}^{2}+\mu \alpha_{1}\|\eta\|_{2}^{2}+\mu \alpha_{2}\|\beta\|_{2}^{2} \\
\geq \quad m_{0}\left[\|\operatorname{sym} \nabla v\|_{2}^{2}+\|\operatorname{sym} q\|_{2}^{2}-\theta\|\operatorname{sym} \nabla v\|_{2}^{2}-\frac{1}{\theta}\|\operatorname{sym} q\|_{2}^{2}\right] \quad \text { (Young's inequality) } \\
\quad+\mu L_{c}^{2}\|\operatorname{Curl} q\|_{2}^{2}+\frac{1}{2} \mu \alpha_{1}\|\eta\|_{2}^{2}+\frac{1}{2} \mu \alpha_{2}\|\beta\|_{2}^{2} \\
\quad+\frac{1}{2} \mu \alpha_{1}\|\operatorname{sym} q\|_{2}^{2}+\frac{1}{2} \mu \alpha_{2}\|\operatorname{skew} q\|_{2}^{2} \quad(\text { using }\|\operatorname{sym} q\| \leq \eta, \| \text { skew } q \| \leq \beta) \\
=m_{0}(1-\theta)\|\operatorname{sym} \nabla v\|_{2}^{2}+\left[m_{0}\left(1-\frac{1}{\theta}\right)+\frac{1}{2} \mu \alpha_{1}\right]\|\operatorname{sym} q\|_{2}^{2}+\frac{1}{2} \mu \alpha_{2} \| \text { skew } q \|_{2}^{2} \\
\quad+\mu L_{c}^{2}\|\operatorname{Curl} q\|_{2}^{2}+\frac{1}{2} \mu \alpha_{1}\|\eta\|_{2}^{2}+\frac{1}{2} \mu \alpha_{2}\|\beta\|_{2}^{2} .
\end{gathered}
$$

So, choosing $\theta$ such that $\frac{m_{0}}{m_{0}+\frac{1}{2} \mu \alpha_{1}}<\theta<1$, and using Korn's first inequality (see e.g. [84]), there exists some positive constant $C\left(m_{0}, \mu, \alpha_{1}, \alpha_{2}, L_{c}, \Omega\right)>0$ such that

$$
a(z, z) \geq C\left[\|v\|_{V}^{2}+\|q\|_{\mathrm{H}(\mathrm{Curl} ; \Omega)}^{2}+\|\eta\|_{2}^{2}+\|\beta\|_{2}^{2}\right]=C\|z\|_{Z}^{2} \quad \forall z=(v, q, \eta, \beta) \in \mathrm{W} .
$$

Hence, we get from Theorem 4.1 the existence of a solution for the weak formulation (4.4) with $\alpha_{2}>0$ (and $\alpha_{1}>0$ ).

\subsection{Uniqueness of the strong solution}

If in the geometrically linear classical plasticity model with isotropic hardening, the uniqueness of the weak solution is obtained from the formulation in a variational inequality (see [60, Theorem 7.3]) the uniqueness of the weak solution in the context of gradient plasticity with isotropic hardening has not yet been completely established. However, in some particular cases, the uniqueness has been obtained provided weak solutions are regular enough (see e.g. [60, pp.210212]).

The diffculty here is that the coerciviy of the bilinear form $\boldsymbol{a}$, which is key to get the uniqueness of the solution of the weak formulation, is only obtained on the closed convex cone $\mathrm{W}$ 
and not on the entire space Z. Therefore, one cannot use the standard argument of involving the difference $w_{1}-w_{2}$ of two solutions $w_{1}$ and $w_{2}$ and getting the uniqueness, since that difference does not always belong to the closed convex cone W. We recall that, for Prager-type linear kinematical hardening, the uniqueness of strong solutions in infinitesimal perfect gradient plasticity was established in [86]. In our context, we will prove in the next theorem that requiring Curl Curl $p \in \mathrm{H}^{1}\left(\left[(0, T] ; \mathrm{L}^{2}\left(\Omega, \mathbb{R}^{3 \times 3}\right)\right)\right.$ is enough to guarantee the uniqueness of the strong solution.

Theorem 4.3 Let $w=\left(u, \Gamma_{p}\right)$ be a solution of the weak formulation (4.4) obtained in Theorem 4.2 with $\Gamma_{p}=\left(p, \gamma_{p}, \omega_{p}\right)$. If $p$ satisfies $\operatorname{Curl}$ Curl $p \in \mathrm{H}^{1}\left([0, T] ; \mathrm{L}^{2}\left(\Omega, \mathbb{R}^{3 \times 3}\right)\right)$, then $w$ is the unqiue strong solution, i.e., the unique solution of the strong formulation in Section 4.1.

Proof: In fact, we first notice that if $w=\left(u, \Gamma_{p}\right) \in \mathrm{W}$ is a solution of $(4.4)$ with $\Gamma_{p}=\left(p, \gamma_{p}, \omega_{p}\right)$ and Curl Curl $p \in L^{2}\left(\Omega, \mathbb{R}^{3 \times 3}\right)$, then choosing appropriately test functions and integrating by parts, we easily get that $w=\left(u, \Gamma_{p}\right)$ satisfies the equilibrium equation (3.1) on the one hand and $\Gamma_{p}$ satisfies the flow rule in dual form

$$
\left\langle\dot{\Gamma}_{p}, \bar{\Sigma}-\Sigma_{p}\right\rangle \leq 0 \quad \forall \bar{\Sigma}
$$

on the other hand.

Let us now consider two solutions $w_{i}=\left(u_{i}, \Gamma_{p_{i}}\right) \quad i=1,2$ of (4.4) with $\Gamma_{p_{i}}=\left(p_{i}, \gamma_{p_{i}}, \omega_{p_{i}}\right)$, satisfying the same initial conditions and let $\Sigma_{p_{i}}=\left(\Sigma_{E_{i}}, g_{1_{i}}, g_{2_{i}}\right)$ be the corresponding stresses. That is,

$$
\Sigma_{E_{i}}=\sigma_{i}-\mu L_{c}^{2} \text { Curl Curl } p_{i}, \quad g_{1_{i}}=-\mu \alpha_{1} \gamma_{p_{i}}, \quad g_{2_{i}}=-\mu \alpha_{2} \omega_{p_{i}} .
$$

Hence, $\Gamma_{p_{i}}$ and $\Sigma_{p_{i}}$ satisfy (4.14), that is,

$$
\left\langle\dot{\Gamma}_{p_{1}}, \bar{\Sigma}-\Sigma_{p_{1}}\right\rangle \leq 0 \quad \text { and } \quad\left\langle\dot{\Gamma}_{p_{2}}, \bar{\Sigma}-\Sigma_{p_{2}}\right\rangle \leq 0 \quad \forall \bar{\Sigma}
$$

Now choose $\bar{\Sigma}=\Sigma_{p_{2}}$ in $(4.16)_{1}$ and $\bar{\Sigma}=\Sigma_{p_{1}}$ in (4.16) $)_{2}$ and add up to get

$$
\left\langle\Sigma_{p_{2}}-\Sigma_{p_{1}}, \dot{\Gamma}_{p_{1}}-\dot{\Gamma}_{p_{2}}\right\rangle \leq 0
$$

That is

$$
\begin{aligned}
& \left\langle\sigma_{2}-\sigma_{1}, \dot{p}_{1}-\dot{p}_{2}\right\rangle+\mu L_{c}^{2}\left\langle\operatorname{Curl} \operatorname{Curl}\left(p_{2}-p_{1}\right), \dot{p}_{2}-\dot{p}_{1}\right\rangle \\
& +\mu \alpha_{1}\left(\gamma_{p_{2}}-\gamma_{p_{1}}\right)\left(\dot{\gamma}_{p_{2}}-\dot{\gamma}_{p_{1}}\right)+\mu \alpha_{2}\left(\omega_{p_{2}}-\omega_{p_{1}}\right)\left(\dot{\omega}_{p_{2}}-\dot{\omega}_{p_{1}}\right) \leq 0 .
\end{aligned}
$$

Since $\sigma$ is symmetric, the latter is equivalent to

$$
\begin{aligned}
\left\langle\sigma_{2}-\sigma_{1}\right. & \left.\operatorname{sym}\left(\dot{p}_{1}-\dot{p}_{2}\right)\right\rangle+\mu L_{c}^{2}\left\langle\operatorname{Curl} \operatorname{Curl}\left(p_{2}-p_{1}\right), \dot{p}_{2}-\dot{p}_{1}\right\rangle \\
& +\mu \alpha_{1}\left(\gamma_{p_{2}}-\gamma_{p_{1}}\right)\left(\dot{\gamma}_{p_{2}}-\dot{\gamma}_{p_{1}}\right)+\mu \alpha_{2}\left(\omega_{p_{2}}-\omega_{p_{1}}\right)\left(\dot{\omega}_{p_{2}}-\dot{\omega}_{p_{1}}\right) \leq 0 .
\end{aligned}
$$

Now, substitute $\operatorname{sym} p_{i}=\operatorname{sym}\left(\nabla u_{i}\right)-\mathbb{C}^{-1} \sigma_{i}$ obtained from the elasticity relation, into equation (4.19) and get

$$
\begin{gathered}
\left\langle\sigma_{2}-\sigma_{1}, \mathbb{C}^{-1}\left(\dot{\sigma}_{2}-\dot{\sigma}_{1}\right)\right\rangle+\mu L_{c}^{2}\left\langle\operatorname{Curl} \operatorname{Curl}\left(p_{2}-p_{1}\right), \dot{p}_{2}-\dot{p}_{1}\right\rangle+\mu \alpha_{1}\left(\gamma_{p_{2}}-\gamma_{p_{1}}\right)\left(\dot{\gamma}_{p_{2}}-\dot{\gamma}_{p_{1}}\right) \\
+\mu \alpha_{2}\left(\omega_{p_{2}}-\omega_{p_{1}}\right)\left(\dot{\omega}_{p_{2}}-\dot{\omega}_{p_{1}}\right) \leq\left\langle\sigma_{1}-\sigma_{2}, \operatorname{sym}\left(\nabla \dot{u}_{1}\right)-\operatorname{sym}\left(\nabla \dot{u}_{2}\right)\right\rangle .
\end{gathered}
$$


Now, notice that from the equilibrium equation we get

$$
\int_{\Omega}\left\langle\sigma_{1}-\sigma_{2}, \operatorname{sym}\left(\nabla \dot{u}_{1}\right)-\operatorname{sym}\left(\nabla \dot{u}_{2}\right)\right\rangle d x=0 .
$$

Hence, for a.e. $t \in(0, T)$, integrate (4.20) over $\Omega \times(0, t)$ then after integrating the term with Curl Curl by parts, we get

$$
\begin{aligned}
\int_{0}^{t} \frac{d}{d s}\left[\left\|\mathbb{C}^{-1 / 2}\left(\sigma_{2}(s)-\sigma_{1}(s)\right)\right\|_{2}^{2}+\mu L_{c}^{2}\left\|\operatorname{Curl}\left(p_{1}(s)-p_{2}(s)\right)\right\|_{2}^{2}\right. \\
\left.\quad+\mu \alpha_{1}\left\|\gamma_{p_{2}}(s)-\gamma_{p_{1}}(s)\right\|_{2}^{2}+\mu \alpha_{2}\left\|\omega_{p_{1}}(s)-\omega_{p_{2}}(s)\right\|_{2}^{2}\right] d s \leq 0 .
\end{aligned}
$$

Therefore, we obtain

$$
\begin{aligned}
\left\|\left(\mathbb{C}^{-1}\right)^{1 / 2}\left(\sigma_{2}-\sigma_{1}\right)\right\|_{2}^{2}+ & \mu L_{c}^{2}\left\|\operatorname{Curl}\left(p_{1}-p_{2}\right)\right\|_{2}^{2} \\
& +\mu \alpha_{1}\left\|\gamma_{p_{2}}-\gamma_{p_{1}}\right\|_{2}^{2}+\mu \alpha_{2}\left\|\omega_{p_{2}}-\omega_{p_{1}}\right\|_{2}^{2}=0,
\end{aligned}
$$

from which we get $\sigma_{1}=\sigma_{2}, \operatorname{Curl} p_{1}=\operatorname{Curl} p_{2}, \gamma_{p_{1}}=\gamma_{p_{2}}, \omega_{p_{2}}=\omega_{p_{1}}$ and hence, $\Sigma_{E_{1}}=\Sigma_{E_{2}}$.

Now, let us prove that $p_{1}=p_{2}$. In fact, from the definition of the normal cone it follows that $\dot{p}_{i}=0$ and $\dot{\gamma}_{p_{i}}=\dot{\omega}_{p_{i}}=0$ inside the elastic domain $\mathcal{E}$, which from the initial conditions imply that $p_{i}=0$ inside $\mathcal{E}$. Now, looking at the flow rule in dual form in Table 2 , we easily obtain that $\operatorname{sym} \dot{p}_{1}=\operatorname{sym} \dot{p}_{2}$ and skew $\dot{p}_{1}=$ skew $\dot{p}_{2}$ on each surface $S_{k}$. Therefore, $\dot{p}_{1}=\dot{p}_{2}$ which implies that $p_{1}=p_{2}$ from the initial conditions.

In order to show that $u_{1}=u_{2}$, we use $\operatorname{sym}\left(\nabla u_{i}\right)=\mathbb{C}^{-1} \sigma_{i}+\operatorname{sym} p_{i}$ obtained from the elasticity relation and get

$$
\operatorname{sym}\left(\nabla\left(u_{1}-u_{2}\right)\right)=\mathbb{C}^{-1}\left(\sigma_{1}-\sigma_{2}\right)+\operatorname{sym}\left(p_{1}-p_{2}\right)=0,
$$

and hence, from the first Korn's inequality (see e.g. [84]), we get $\nabla\left(u_{1}-u_{2}\right)=0$ which implies that $u_{1}=u_{2}$. Therefore, we finally obtain

$$
u_{1}=u_{2}, \quad \sigma_{1}=\sigma_{2}, \quad p_{1}=p_{2}, \quad \gamma_{p_{1}}=\gamma_{p_{2}}, \quad \omega_{p_{1}}=\omega_{p_{2}},
$$

and thus the uniqueness of a strong solution to the mathematical problem describing our model of rate-independent geometrically linear gradient plasticity with isotropic hardening and plastic spin in the case $\alpha_{2}>0$, where there is always isotropic hardening in the spin-evolution equation.

\subsection{Perfect gradient plasticity with spin}

Inspection of the uniqueness proof for strong solutions in Section 4.4 shows that in the case with zero isotropic plastic strain and spin hardening, and the homogeneous boundary conditions $\left.u\right|_{\Gamma}=0$ and $p \times\left. n\right|_{\Gamma}=0$, elastic stresses $\sigma$, elastic strains $\varepsilon_{e}=\operatorname{sym} e$ and furthermore elastic distortions $e=\nabla u-p$ are unique. The uniqueness with respect to elastic distortions uses again the new Korn's inequality for incompatible tensor fields [94] since $e \times\left. n\right|_{\Gamma}=0$. In this case, the extra inclusion of the spin and the dislocation density tensor allow to improve uniqueness from elastic strains to elastic distortions. Notice that, in the context of crystal gradient plasticity, non-uniqueness has been shown in [13] for the case of nonhomogeneous displacement boundary conditions, focussing on the simple shear of a constrained strip endowed with multiple slip systems. The same type of non-unqiueness results have been obtained also in $[46,107,104]$. 


\section{The complete mathematical formulation in the no-spin-hardening case}

Here we set $\alpha_{2}=0$ in the free-energy $\Psi$ in (3.4)-(3.5). The case $\alpha_{2}=0$ means that there is no isotropic hardening in the spin-evolution. At present we believe that it is this case which deserves special attention, since in this model we extend classical plasticity in the weakest possible way to depend on plastic spin. Notably, we do not incur additional spin-hardening. The dissipation function $\Delta$ is still the same given in (3.21) and the yield function is given in (4.1). Also, in this model the influence of the SSD's and GND's on plastic flow is neatly separated: the SSD-distribution influences only isotropic hardening through the classical mechanism and the GND-distribution determines the nonlocal kinematic hardening.

\subsection{The strong formulation of the model}

The strong formulation in the no-spin-hardening case is obtained exactly as in Section 4.1. For the clarity of exposition, we chose to present here the whole formulation summarized in Table 3 below, instead of just pointing out the differences w.r.t. Table 2.

\subsection{The weak formulation of the model}

Also, following Section 4.2, we derive the weak formulation of the model in the no-spin-hardening case as the variational inequality

$$
\begin{aligned}
& \int_{\Omega}\left\langle\mathbb{C}_{\text {iso }}(\operatorname{sym} \nabla u-\operatorname{sym} p),(\operatorname{sym} \nabla v-\operatorname{sym} q)-(\operatorname{sym} \nabla \dot{u}-\operatorname{sym} \dot{p})\right\rangle d x+\alpha_{1} \mu \int_{\Omega} \gamma_{p}\left(\eta-\dot{\gamma}_{p}\right) d x \\
& \quad+\mu L_{c}^{2} \int_{\Omega}\langle\operatorname{Curl} p, \operatorname{Curl}(q-\dot{p})\rangle d x+\int_{\Omega} \Delta(\bar{\Gamma}) d x-\int_{\Omega} \Delta\left(\dot{\Gamma}_{p}\right) d x \geq \int_{\Omega} f(v-\dot{u}) d x
\end{aligned}
$$

\subsection{Existence result in the no-spin-hardening case}

As in Section 4.3, the existence result in the no-spin-hardening is also obtained through the abstract result in Theorem 4.1. The functionals $j$ and $\ell$ remain as in (4.8) and (4.9) respectively, the bilinear form $\boldsymbol{a}$ in this case is defined as

$$
\begin{gathered}
\boldsymbol{a}(w, z)=\int_{\Omega}\left[\left\langle\mathbb{C}_{\text {iso }}(\operatorname{sym} \nabla u-\operatorname{sym} p), \operatorname{sym} \nabla v-\operatorname{sym} q\right)\right\rangle \\
\left.+\mu L_{c}^{2}\langle\operatorname{Curl} p, \operatorname{Curl} q\rangle+\mu \alpha_{1} \gamma_{p} \eta\right] d x, \\
\forall w=\left(u, p, \gamma_{p}, \omega_{p}\right), z=(v, q, \eta, \beta) \in \mathbf{Z} .
\end{gathered}
$$

The existence result for the weak formulation in the no-spin-hardening case is obtained in the following theorem.

Theorem 5.1 The weak formulation (5.1) in the no-spin-hardening case (i.e., $\alpha_{2}=0$ ) written as: find $w=\left(u, p, \gamma_{p}, \omega_{p}\right) \in \mathrm{H}^{1}(0, T ; Z)$ such that $w(0)=0$ and $\dot{w}(t) \in \mathrm{W}$ for a.e. $t \in[0, T]$

$$
\boldsymbol{a}(\dot{w}, z-w)+j(z)-j(\dot{w}) \geq\langle\ell, z-\dot{w}\rangle \text { for every } z \in \mathbf{Z} \text { and for a.e. } t \in[0, T]
$$




\begin{tabular}{|c|c|}
\hline $\begin{array}{l}\text { Additive split of distortion: } \\
\text { Equilibrium: }\end{array}$ & $\begin{array}{l}\nabla u=e+p, \quad \varepsilon_{e}=\operatorname{sym} e, \quad \varepsilon_{p}=\operatorname{sym} p \\
\operatorname{Div} \sigma+f=0 \text { with } \sigma=\mathbb{C}_{\mathrm{iso}} \varepsilon_{e}\end{array}$ \\
\hline Free energy: & $\frac{1}{2}\left\langle\mathbb{C}_{\mathrm{iso}} \varepsilon_{e}, \varepsilon_{e}\right\rangle+\frac{1}{2} \mu L_{c}^{2}\|\operatorname{Curl} p\|^{2}+\frac{1}{2} \mu \alpha_{1}\left|\gamma_{p}\right|^{2}$ \\
\hline $\begin{array}{l}\text { Yield condition: } \\
\text { where }\end{array}$ & $\begin{array}{l}\phi\left(\Sigma_{p}\right)=0 \text { with } \phi \text { given in }(4.1) \\
\Sigma_{p}=\left(\Sigma_{E}, g_{1}, g_{2}\right), \quad \Sigma_{E}:=\sigma+\Sigma_{\text {curl }}^{\text {lin }}, \quad \Sigma_{\text {curl }}=-\mu L_{c}^{2} \text { Curl Curl } p \\
g_{1}=-\mu \alpha_{1} \gamma_{p}, \quad g_{2}=0\end{array}$ \\
\hline $\begin{array}{l}\text { Dissipation inequality: } \\
\text { Dissipation function: } \\
\text { Flow law in primal form: }\end{array}$ & $\begin{array}{l}\int_{\Omega}\left[\left\langle\operatorname{dev} \operatorname{sym} \Sigma_{E}, \operatorname{sym} \dot{p}\right\rangle+\left\langle\text { skew } \Sigma_{E}, \text { skew } \dot{p}\right\rangle+g_{1} \dot{\gamma}_{p}\right] d x \geq 0 \\
\Delta\left(\dot{\Gamma}_{p}\right) \text { is defined in }(3.21) \\
\Sigma_{p} \in \partial \Delta\left(\dot{\Gamma}_{p}\right)\end{array}$ \\
\hline Flow law in dual form on $\mathcal{S}_{1}$ : & $\begin{cases}\operatorname{sym} \dot{p}=\lambda \frac{\operatorname{dev} \operatorname{sym} \Sigma_{E}}{\left\|\operatorname{dev} \operatorname{sym} \Sigma_{E}\right\|}, & \dot{\gamma}_{p}=\lambda=\|\operatorname{sym} \dot{p}\| \\
\text { skew } \dot{p}=0, & \dot{\omega}_{p}=0\end{cases}$ \\
\hline Flow law in dual form on $\mathcal{S}_{2}$ : & 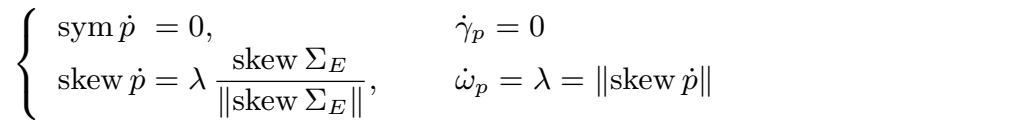 \\
\hline Flow law in dual form on $\mathcal{S}_{3}$ : & $\left\{\begin{aligned} \operatorname{sym} \dot{p} & =\frac{2 \lambda}{\sigma_{0}^{2}}\left(\left\|\operatorname{dev} \operatorname{sym} \Sigma_{E}\right\|+g_{1}\right) \frac{\operatorname{dev} \operatorname{sym} \Sigma_{E}}{\left\|\operatorname{dev} \operatorname{sym} \Sigma_{E}\right\|} \\
\text { skew } \dot{p} & =\frac{2 \lambda}{\widehat{\sigma}_{0}^{2}} \text { skew } \Sigma_{E} \\
\dot{\gamma}_{p} & =\frac{2 \lambda}{\sigma_{0}^{2}}\left(\| \text { dev sym } \Sigma_{E} \|+g_{1}\right)=\|\operatorname{sym} \dot{p}\| \\
\dot{\omega}_{p} & =\frac{2 \lambda}{\widehat{\sigma}_{0}^{2}} \| \text { skew } \Sigma_{E}\|=\| \text { skew } \dot{p} \| \\
\|\dot{p}\| \quad & \sqrt{\dot{\gamma}_{p}^{2}+\dot{\omega}_{p}^{2}}, \quad \lambda=\frac{1}{2} \sqrt{\sigma_{0}^{2} \dot{\gamma}_{p}^{2}+\widehat{\sigma}_{0}^{2} \dot{\omega}_{p}^{2}}\end{aligned}\right.$ \\
\hline $\begin{array}{l}\text { KKT conditions: } \\
\text { Boundary conditions for } p \text { : } \\
\text { Function space for } p:\end{array}$ & $\begin{array}{l}\lambda \geq 0, \quad \phi\left(\Sigma_{E}, g_{1}, 0\right) \leq 0, \quad \lambda \phi\left(\Sigma_{E}, g_{1}, 0\right)=0 \\
p \times n=0 \text { on } \Gamma, \quad(\text { Curl } p) \times n=0 \text { on } \partial \Omega \backslash \Gamma \\
p(t, \cdot) \in \mathrm{H}\left(\text { Curl } ; \Omega, \mathbb{R}^{3 \times 3}\right)\end{array}$ \\
\hline
\end{tabular}

Table 3: The model with isotropic hardening only in the plastic strain-evolution (the case $\alpha_{2}=0$ ). Notice that the boundary conditions on $p$ necessitates at least $p \in \mathrm{H}\left(\mathrm{Curl} ; \Omega, \mathbb{R}^{3 \times 3}\right)$, which is not guaranteed looking at the free-energy and the dissipation function. However, this will be obtained from a new Korn's type inequality for incompatible tensor fields derived by Neff et al. in [91, 92, 93, 94] .

with $\boldsymbol{a}$ defined in (5.2) and $j$ and $\ell$ defined in (4.8)-(4.9), has a solution for some suitable Hilbert space $\mathbf{Z}$ and some closed convex cone $\mathrm{W}$ in $\mathrm{Z}$.

Proof: First of all, notice that since the bilinear form $\boldsymbol{a}$ does not contain explicitly the variable $\beta$, it is impossible to derive the coercivity of the bilinear form in any normed space in all the 
variables $v, q, \eta$ and $\beta$. Therefore, we are not in a position to apply directly the abstract result in Theorem 4.1. The new solution strategy here for the existence result is to first find $u, p$ and $\gamma_{p}$, and construct $\omega_{p}$ a posteriori. To this end, we define

$$
\Delta_{0}(q, \eta):= \begin{cases}D(\|\operatorname{sym} q\|, \| \text { skew } q \|) & \text { if }\|\operatorname{sym} q\| \leq \eta, \\ \infty & \text { otherwise }\end{cases}
$$

where we recall that

$$
D(s, t):=\sqrt{\sigma_{0}^{2} s^{2}+\widehat{\sigma}_{0}^{2} t^{2}} .
$$

We then reformulate the problem as follows: find $w=\left(u, p, \gamma_{p}\right) \in \mathrm{H}^{1}(0, T ; \mathrm{Z})$ such that $w(0)=0$, $\dot{w}(t) \in \mathrm{W}$ for a.e. $t \in[0, T]$

$$
\boldsymbol{a}(\dot{w}, z-w)+j_{0}(z)-j_{0}(\dot{w}) \geq\langle\ell, z-\dot{w}\rangle \text { for every } z=(v, q, \eta) \in \mathrm{W} \text { and for a.e. } t \in[0, T],
$$

where we let

$$
\begin{aligned}
& j_{0}(z):= \begin{cases}\int_{\Omega} \Delta_{0}(q, \eta) d x & \text { if } z=(v, q, \eta) \in \mathrm{W} \\
\infty & \text { otherwise, }\end{cases} \\
& \mathrm{Z}=\mathrm{V} \times \mathrm{Q} \times \Lambda, \\
& \mathrm{W}=\{z=(v, q, \eta) \in \mathrm{Z} \mid\|\operatorname{sym} q\| \leq \eta \text { a.e. in } \Omega\}, \\
& \mathrm{V}=\mathrm{H}_{0}^{1}\left(\Omega, \Gamma_{\mathrm{D}}, \mathbb{R}^{3}\right)=\left\{v \in \mathrm{H}^{1}\left(\Omega, \mathbb{R}^{3}\right) \mid v_{\mid \Gamma}=0\right\}, \\
& \mathrm{Q}=\mathrm{H}_{0}(\operatorname{Curl} ; \Omega, \Gamma, \mathfrak{s l}(3)) \quad \text { defined in }(2.7), \\
& \Lambda=L^{2}(\Omega),
\end{aligned}
$$

equipped with the norms

$$
\begin{aligned}
& \|v\|_{V}:=\|\nabla v\|_{L^{2}}, \quad\|q\|_{Q}:=\|q\|_{\mathrm{H}(\operatorname{Curl} ; \Omega)}, \\
& \|z\|_{Z}^{2}:=\|v\|_{V}^{2}+\|q\|_{Q}^{2}+\|\eta\|_{L^{2}}^{2} \quad \text { for } z=(v, q, \eta) \in \mathrm{Z} .
\end{aligned}
$$

Now, for the existence of a solution to the problem (5.6) following Theorem 4.1), we only need to check that the bilinear form $\boldsymbol{a}$ is coercive in W. Following the coercivity inequality obtained in (4.13), we immediately get a positive constant $C=C\left(m_{0}, \mu, \alpha_{1}, L_{c}, \Omega\right)>0$ such that

$$
a(z, z) \geq C\left[\|v\|_{V}^{2}+\|\operatorname{sym} q\|^{2}+\|\operatorname{Curl} q\|_{2}^{2}+\|\eta\|_{2}^{2}\right] .
$$

But this estimate is not enough to establish coercivity. Indeed, the skew-symmetric (spin) part skew $q$ of $q$ is not controlled locally.

Motivated by the well-posedness question for precursors to this model [88, 28], Neff et al. [91, 92, 93, 94], derived a new inequality extending Korn's first inequality to incompatible tensor fields, namely there exists a constant $C(\Omega)>0$ such that

$$
\begin{aligned}
\forall p \in \mathrm{H}\left(\mathrm{Curl} ; \Omega, \mathbb{R}^{3 \times 3}\right), \quad p \times\left. n\right|_{\Gamma}=0: & \underbrace{\|p\|_{L^{2}(\Omega)}}_{\text {plastic distortion }} \leq C(\Omega)(\underbrace{\|\operatorname{sym} p\|_{L^{2}(\Omega)}}_{\text {plastic strain }}+\underbrace{\|\operatorname{Curl} p\|_{L^{2}(\Omega)}}_{\text {dislocation density }}) .
\end{aligned}
$$


This shows that if we consider the closure $\mathrm{H}_{\text {sym }}(\mathrm{Curl}, \Omega, \Gamma ; \mathfrak{s l}(3))$ of the linear subspace

$$
\left\{q \in C^{\infty}\left(\bar{\Omega}, \mathbb{R}^{3 \times 3}\right) \mid \operatorname{tr} q=0, q \times n=0 \text { on } \Gamma\right\}
$$

in the norm

$$
\|q\|_{\text {sym, curl }}^{2}:=\|\operatorname{sym} q\|_{L^{2}}^{2}+\|\operatorname{Curl} q\|_{L^{2}}^{2},
$$

then we have the decisive identity

$$
\mathrm{H}_{\mathrm{sym}}(\operatorname{Curl}, \Omega, \Gamma ; \mathfrak{s l}(3)) \equiv \mathrm{H}_{0}(\operatorname{Curl}, \Omega, \Gamma ; \mathfrak{s l}(3))
$$

with equivalence of norms. Therefore, we have the coercivity inequality

$$
\boldsymbol{a}(z, z) \geq C\left[\|v\|_{V}^{2}+\|q\|_{Q}^{2}+\|\eta\|_{2}^{2}\right]=\|z\|_{Z}^{2} \quad \forall z \in \mathrm{W},
$$

from which we obtain the existence of a solution $\left(u, p, \gamma_{p}\right) \in \mathrm{W}$ to the problem (5.6). Now setting a posteriori

$$
\omega_{p}(t, x):=\int_{0}^{t}\|\operatorname{skew} \dot{p}(s, x)\| d s,
$$

it follows that $\left(u, p, \gamma_{p}, \omega_{p}\right)$ is a solution to the original problem (5.3).

Remark 5.1 Notice again that isotropic hardening in the spin-evolution is not necessary for existence of a solution to the problem and it is not connected to the uniqueness question either. In fact, arguing as in Section 4.4 we get the inequality (4.22) with $\alpha_{2}=0$, from which and from the flow law in dual form on each $\mathcal{S}_{k}$ of the yield surface, we deduce the uniqueness of $u, \sigma, p$ and $\gamma_{p}$ while the uniqueness of $\omega_{p}$ follows from (5.14) and from the uniqueness of $p$. Therefore, the strong solution is unique also in the case where there is no isotropic hardening in the spin-evolution.

\subsection{Is it possible to accommodate the special case $\widehat{\sigma}_{0}=0$ in our model?}

In Gurtin's visco-plastic model [46] it is possible to consider $\widehat{\sigma}_{0}=0$. In our setting, this case corresponds to the dissipation function

$$
\Delta(q, \eta, \beta):= \begin{cases}\sigma_{0}\|\operatorname{sym} q\| & \text { if }\|\operatorname{sym} q\| \leq \eta \quad \text { and } \quad \| \text { skew } q \| \leq \beta \\ \infty & \text { otherwise }\end{cases}
$$

and the elastic region

$$
\mathcal{E}:=\left\{\Sigma_{p}=\left(\Sigma_{E}, g_{1}, g_{2}\right) \mid\left\|\operatorname{dev} \operatorname{sym} \Sigma_{E}\right\|-\sigma_{0}+g_{1} \leq 0 \text { and } \| \text { skew } \Sigma_{E} \|+g_{2} \leq 0\right\} .
$$

The flow law in dual form is given in Table 4 below.

Table 4 illustrates why both initial yield stresses $\sigma_{0}$ and $\widehat{\sigma}_{0}$ have to be strictly positive. In fact, since $g_{2}$ may be zero initially, the elastic domain $\mathcal{E}$ in (5.16) may not have non-empty interior. Therefore, this forbids the use of $\widehat{\sigma}_{0}=0$. In the visco-plastic setting $\widehat{\sigma}_{0}=0$ may be accommodated. 


\begin{tabular}{|c|c|}
\hline Free energy: & $\frac{1}{2}\left\langle\mathbb{C}_{\mathrm{iso}} \varepsilon_{e}, \varepsilon_{e}\right\rangle+\frac{1}{2} \mu L_{c}^{2}\|\operatorname{Curl} p\|^{2}+\frac{1}{2} \mu \alpha_{1}\left|\gamma_{p}\right|^{2}+\frac{1}{2} \mu \alpha_{2}\left|\omega_{p}\right|^{2}$ \\
\hline Elastic region: & $\mathcal{E}:=\left\{\left(\Sigma_{E}, g_{1}, g_{2}\right) \mid\left\|\operatorname{dev} \operatorname{sym} \Sigma_{E}\right\|-\sigma_{0}+g_{1} \leq 0\right.$ and $\|$ skew $\left.\Sigma_{E} \|+g_{2} \leq 0\right\}$ \\
\hline Yield surface: & $\partial \mathcal{E}=\mathcal{S}_{1} \cup \mathcal{S}_{2}$ \\
\hline where & $\mathcal{S}_{1}:=\left\{\left(\Sigma_{E}, g_{1}, g_{2}\right) \mid\left\|\operatorname{dev} \operatorname{sym} \Sigma_{E}\right\|-\sigma_{0}+g_{1}=0\right\}$ \\
\hline & $\mathcal{S}_{2}:=\left\{\left(\Sigma_{E}, g_{1}, g_{2}\right) \mid \|\right.$ skew $\left.\Sigma_{E} \|+g_{2}=0\right\}$ \\
\hline Dissipation function: & $\Delta(q, \eta, \beta):= \begin{cases}\sigma_{0}\|\operatorname{sym} q\| & \text { if }\|\operatorname{sym} q\| \leq \eta \quad \text { and } \quad \| \text { skew } q \| \leq \beta \\
\infty & \text { otherwise }\end{cases}$ \\
\hline$m$ & $\operatorname{sym} \dot{p}=\lambda \frac{\operatorname{dev} \operatorname{sym} \Sigma_{E}}{\left\|\operatorname{dev} \operatorname{sym} \Sigma_{E}\right\|}, \quad$ skew $\dot{p}=0, \quad \dot{\gamma}_{p}=\lambda, \quad \dot{\omega}_{p}=0 \quad$ on $\mathcal{S}_{1}$ \\
\hline & $\operatorname{sym} \dot{p}=0, \quad$ skew $\dot{p}=\lambda \frac{\text { skew } \Sigma_{E}}{\| \text { skew } \Sigma_{E} \|}, \quad \dot{\gamma}_{p}=0, \quad \dot{\omega}_{p}=\lambda \quad$ on $\mathcal{S}_{2}$ \\
\hline
\end{tabular}

Table 4: The flow rule in dual form in the case $\widehat{\sigma}_{0}=0$ and $\alpha_{2}>0$.

\section{The limit case of vanishing characteristic length scale $L_{c} \rightarrow 0$}

In the limit case $L_{c} \rightarrow 0$, looking at the flow rule in its dual formulation, we first observe that the thermodynamic driving stress $\Sigma_{E} \in \mathbb{R}^{3 \times 3}$ reduces to the symmetric Cauchy stress tensor $\sigma \in \operatorname{Sym}(3)$ and we see clearly that we do not have the branch $\mathcal{S}_{2}$ and moreover,

$$
\text { on } \mathcal{S}_{1}: \begin{cases}\operatorname{sym} \dot{p}=\lambda \frac{\operatorname{dev} \sigma}{\|\operatorname{dev} \sigma\|}, & \dot{\gamma}_{p}=\lambda=\|\operatorname{sym} \dot{p}\|, \\ \text { skew } \dot{p}=0, & \dot{\omega}_{p}=0,\end{cases}
$$

while on $\mathcal{S}_{3}$ we get from the rate-explicit dual formulation

$$
\begin{cases}\operatorname{sym} \dot{p}=\frac{2 \lambda}{\sigma_{0}^{2}}\left(\left\|\operatorname{dev} \operatorname{sym} \Sigma_{E}\right\|+g_{1}\right) \frac{\operatorname{dev} \operatorname{sym} \Sigma_{E}}{\left\|\operatorname{dev} \operatorname{sym} \Sigma_{E}\right\|}, & \dot{\gamma}_{p}=\frac{2 \lambda}{\sigma_{0}^{2}}\left(\left\|\operatorname{dev} \operatorname{sym} \Sigma_{E}\right\|+g_{1}\right)=\|\operatorname{sym} \dot{p}\| \\ \text { skew } \dot{p}=\frac{2 \lambda}{\widehat{\sigma}_{0}^{2}}\left(\| \text { skew } \Sigma_{E} \|+g_{2}\right) \frac{\text { skew } \Sigma_{E}}{\| \text { skew } \Sigma_{E} \|}, & \dot{\omega}_{p}=\frac{2 \lambda}{\widehat{\sigma}_{0}^{2}}\left(\| \text { skew } \Sigma_{E} \|+g_{2}\right)=\| \text { skew } \dot{p} \|\end{cases}
$$

in the case $L_{c}>0$ and $\alpha_{2}>0$ and 


$$
\begin{cases}\operatorname{sym} \dot{p}=\frac{2 \lambda}{\sigma_{0}^{2}}\left(\left\|\operatorname{dev} \operatorname{sym} \Sigma_{E}\right\|+g_{1}\right) \frac{\operatorname{dev} \operatorname{sym} \Sigma_{E}}{\left\|\operatorname{dev} \operatorname{sym} \Sigma_{E}\right\|}, & \dot{\gamma}_{p}=\frac{2 \lambda}{\sigma_{0}^{2}}\left(\left\|\operatorname{dev} \operatorname{sym} \Sigma_{E}\right\|+g_{1}\right)=\|\operatorname{sym} \dot{p}\| \\ \text { skew } \dot{p}=\frac{2 \lambda}{\widehat{\sigma}_{0}^{2}} \text { skew } \Sigma_{E}, & \dot{\omega}_{p}=\frac{2 \lambda}{\widehat{\sigma}_{0}^{2}} \| \text { skew } \Sigma_{E}\|=\| \text { skew } \dot{p} \|\end{cases}
$$

in the case $L_{c}>0$ and $\alpha_{2}=0$ that altogether

$$
\text { on } \mathcal{S}_{3}: \begin{cases}\operatorname{sym} \dot{p}=\frac{2 \lambda}{\sigma_{0}} \frac{\operatorname{dev} \sigma}{\|\operatorname{dev} \sigma\|}, & \dot{\gamma}_{p}=\frac{2 \lambda}{\sigma_{0}}=\|\operatorname{sym} \dot{p}\|, \\ \text { skew } \dot{p}=0, & \dot{\omega}_{p}=0 .\end{cases}
$$

Therefore, we obtain for $L_{c} \rightarrow 0$ that all driving stress-tensor quantities are symmetric such that, if $p(0) \in \operatorname{Sym}(3)$, then we will have $p(t) \in \operatorname{Sym}(3)$ along the plastic evolution. In that case, our new model turns into

$$
\dot{\varepsilon}_{p}=\widehat{\lambda} \frac{\operatorname{dev} \sigma}{\|\operatorname{dev} \sigma\|}, \quad \dot{\gamma}_{p}=\widehat{\lambda}=\left\|\dot{\varepsilon}_{p}\right\|,
$$

which is the dual formulation of the flow rule for classical plasticity with isotropic hardening based only on the accumulated equivalent plastic strain $\gamma_{p}=\int_{0}^{t}\left\|\dot{\varepsilon}_{p}\right\| d s$.

For us it is interesting to remark that the evolution of plastic spin in our model is related solely to the energetic length scale $L_{c}>0$.

\section{Conclusions and outlook}

From a modelling perspective, it is not difficult to extend the present model to visco-plasticity. However, the well-posedness result (which we expect to hold) needs to be derived along different methods. Moreover, it would be interesting to treat the dynamic case. Both questions are subject of ongoing work.

Since we did not establish unqualified uniqueness in our model (it hinges on the additional regularity Curl Curl $p \in L^{2}\left(\Omega, \mathbb{R}^{3 \times 3}\right)$ ) it will also be interesting to establish higher regularity provided the data are regular. It remains open whether we really could have non-uniqueness of the weak solutions if regularity is missing. Is the dislocation energy contribution Curl $p \in$ $L^{2}\left(\Omega, \mathbb{R}^{3 \times 3}\right)$ strong enough to prevent non-uniqueness? The question we have to answer is, what least amount of hardening will lead to existence and uniqueness in rate-independent gradient plasticity?

We expect furthermore that a computational implementation suggests itself along the lines of [95]. Attendant to these research perspectives, one should look at simple settings of boundary value problems like anti-plane shear to gain more insight in the response of the model and the new features offered by incorporating plastic spin.

Finally, a major challenge from the mathematical point of view is the replacement of the defect energy in $(3.5)_{2}$ by a more physically realistic term. The one-homogeneous term $\mu L_{c} \|$ Curl $p \|$ 
was proposed in [101] in the context of single crystal gradient plasticity and is summarized in $[60$, p.92] while energies of logarithmic form were used in $[37,14]$. However, such defect energies cannot be adopted in the current mathematical framework and hence, their mathematical treatment needs fundamentally new ideas.

Another interesting challenge is the one of studying possible visco-plastic regularizations of our model through either the classical power-law rate-dependence or the proposal in [103].

\section{Acknowledgements:}

The research of Francois Ebobisse has been supported by the National Research Foundation (NRF) of South Africa through the Incentive Grant for Rated Researchers and the International Centre for Theoretical Physics (ICTP) through the Associateship Scheme. The first draft of this work was written at Essen (Germany) while Francois Ebobisse was visiting the Faculty of Mathematics of the University of Duisburg-Essen.

\section{References}

[1] E.C. Aifantis. On the microstructural origin of certain inelastic models. ASME J. Eng. Mater. Technol., 106:326-330, 1984.

[2] E.C. Aifantis. The physics of plastic deformation. Int. J. Plasticity, 3:211-247, 1987.

[3] E.C. Aifantis. On the role of gradients in the localization of deformation and fracture. Int. J. Engrg. Sci., 30:1279-1299, 1992.

[4] E.C. Aifantis. Gradient Plasticity, in Handbook of Materials Behavior Models, Ed. J. Lemaitre, pp. 281-297, Academic Press, New York, 2001.

[5] E.C. Aifantis. Update on a class of gradient theories. Mechanics of Materials, 35:259-280, 2003.

[6] E.C. Aifantis. Gradient material mechanics: Perspectives and prospects. Acta Mech., 225:999-1012, 2014.

[7] H.D. Alber. Materials with Memory. Initial-Boundary Value Problems for Constitutive Equations with Internal Variables. volume 1682 of Lecture Notes in Mathematics. Springer, Berlin, 1998.

[8] L. Anand, M.E. Gurtin, B.D. Reddy. The stored energy of cold work, thermal annealing, and other thermodynamic issues in single crystal plasticity at small length scales. Int. J. Plasticity, 64:1-25, 2015.

[9] L. Bardella. A deformation theory of strain gradient crystal plasticity that accounts for geometrically necessary dislocations. J. Mech. Phys. Solids, 54:128-160, 2006.

[10] L. Bardella. Some remarks on the strain gradient crystal plasticity modelling, with particular reference to the material length scale involved. Int. J. Plasticity, 23:296-322, 2007.

[11] L. Bardella. A comparison between crystal and isotropic strain gradient plasticity theories with accent on the role of the plastic spin. Eur. J. Mech. A/Solids, 28(3):638-646, 2009.

[12] L. Bardella. Size effects in phenomenological strain gradient plasticity constitutively involving the plastic spin. Int. J. Eng. Sci. 48(5):550-568, 2010.

[13] L. Bardella, A. Giacomini. Influence of material parameters and crystallography on the size effects describable by means of strain gradient plasticity. J. Mech. Phys. Solids. 56:2906-2934, 2008.

[14] L. Bardella, A. Panteghini. Modelling the torsion of thin metal wires by distortion gradient plasticity. J. Mech. Phys. Solids. 78:467-492, 2015.

[15] S. Bargmann, B.D. Reddy, B. Klusemann. A computational study of a model of single-crystal strain gradient viscoplasticity with a fully-interactive hardening relation. Int. J. Solids Structures. 51(15-16):2754-2764, 2014.

[16] S. Bauer, P. Neff, D. Pauly, G. Starke. New Poincaré-type inequalities, Comptes Rendus Math. 352(4):163$166,2014$. 
[17] S. Bauer, P. Neff, D. Pauly, G. Starke. Dev-Div-and DevSym-devCurl-inequalities for incompatible square square tensor fields with mixed boundary conditions. ESAIM Control Optim. Calc. Var., 22(1):112-133, 2016.

[18] V.L. Berdichevsky, L.I. Sedov. Dynamic theory of continuously distributed dislocations. Its relation to plasticity theory. PMM, 31(6):981-1000, (1967) (English translation: J. Appl. Math. Mech. (PMM), 9891006, (1967))

[19] V.L. Berdichevsky. Continuum theory of dislocations revisited, Cont. Mech. Thermod., 18:195-222, 2006.

[20] C. Carstensen, K. Hackl, A. Mielke. Non-convex potentials and microstructures in finite-strain plasticity, Proc. Roy. Soc. London Ser. A. 458:299-317, 2002.

[21] M. Chiricotto, L. Giacomelli, G. Tomassetti. Dissipative scale effects in strain-gradient plasticity: the case of simple shear. SIAM J. Appl. Maths., 76(2):688-704, 2016.

[22] Y.F. Dafalias. The plastic spin. J. Appl. Mech.. 52:865-871, 1985.

[23] G. Dal Maso, A. De Simone, M.G. Mora. Quasistatic evolution problems for linearly elastic - perfectly plastic material. Arch. Ration. Mech. Anal.. 180:237-291, 2006.

[24] R. De Wit. A view of the relation between the continuum theory of lattice defects and non-Euclidean geometry in the linear approximation. Int. J. Engng. Sci., 19:1475-1506, 1981.

[25] J.K. Djoko, F. Ebobisse, A.T. McBride, B.D. Reddy. A discontinuous Galerkin formulation for classical and gradient plasticity. Part 1: Formulation and analysis. Comput. Methods Appl. Mech. Engrg.. 196:3881-3897, 2007.

[26] J.K. Djoko, F. Ebobisse, A.T. McBride, B.D. Reddy. A discontinuous Galerkin formulation for classical and gradient plasticity. Part 2: Algorithms and numerial analysis. Comput. Methods Appl. Mech. Engrg., 197:1-22, 2007.

[27] F. Ebobisse, A.T. McBride, B.D. Reddy. On the mathematical formulations of a model of gradient plasticity, in ed. B.D. Reddy, IUTAM-Symposium on Theoretical, Modelling and Computational Aspects of Inelastic Media (in Cape Town, 2008). 117-128. Springer, Berlin, 2008.

[28] F. Ebobisse, P. Neff. Existence and uniqueness in rate-independent infinitesimal gradient plasticity with isotropic hardening and plastic spin. Math. Mech. Solids, 15:691-703, 2010.

[29] F. Ebobisse, P. Neff, E.C. Aifantis. Existence result for a dislocation based model of single crystal gradient plasticity with isotropic or linear kinematic hardening. Quart. J. Mech. Appl. Math., 71:99-124, 2018.

[30] F. Ebobisse, P. Neff, S. Forest. Well-posedness for the microcurl model in both single and polycrystal gradient plasticity. Int. J. Plasticity, 107:1-26, 2018.

[31] F. Ebobisse, P. Neff, B.D. Reddy. Existence results in dislocation based rate-independent isotropic gradient plasticity with kinematical hardening and plastic spin: The case with symmetric local backstress. http://arxiv.org/pdf/1504.01973.pdf.

[32] F. Ebobisse, P. Neff. A fourth order gauge-invariant gradient plasticity model for polycrystals based on Kröner's incompatibility tensor. http://arxiv.org/pdf/1706.08770.pdf, in review.

[33] N.A. Fleck, J.W. Hutchinson. Strain gradient plasticity. Advances in applied mechanics, J.W. Hutchinson and T.Y. Wu (Eds), 33:295-361, 1997.

[34] N.A. Fleck, J.W. Hutchinson. A reformulation of strain gradient plasticity. J. Mech. Phys. Solids, 49:22452271, 2001.

[35] N.A. Fleck, J.R. Willis. A mathematical basis for strain-gradient plasticity theory. Part I: scalar plastic multiplier. J. Mech. Phys. Solids, 57:161-177, 2009.

[36] N.A. Fleck, J.R. Willis. A mathematical basis for strain-gradient plasticity theory. Part II: Tensorial plastic multiplier. J. Mech. Phys. Solids, 57:1045-1057, 2009.

[37] S. Forest., N. Guéninchault. Inspection of free-energy functions in gradient crystal plasticity. Acta Mech. Sinica, 29:763-772, 2013.

[38] G. Francfort, A. Giacomini. Small strain heterogenous elastoplasticity revisited. Comm. Pure Appl. Math., 65(9):1185-1241, 2012. 
[39] G. Francfort, A. Giacomini, J. Marigo. The elasto-plasticity exquisite corpse: A Suquet legacy. J. Mech. Phys. Solids, i97:125-139, 2016.

[40] H. Gao, Y. Huang, W.D. Nix, J.W. Hutchinson. Mechanism-based strain gradient plasticity-I. Theory. J. Mech. Phys. Solids, 47:1239-1263, 1999.

[41] I.-D. Ghiba, P. Neff, A. Madeo, I. Münch. A variant of the linear isotropic indeterminate couple stress model with symmetric local force-stress, symmetric nonlocal force-stress, symmetric couple-stresses and complete traction boundary conditions. Math. Mech. Solids. 22(6):1221-1266, 2017.

[42] A. Giacomini. On the energetic formulation of the Gurtin and Anand model in strain gradient plasticity. Discrete Contin. Dyn. Syst. Ser. B, 17:527-552, 2012.

[43] A. Giacomini, L. Lussardi. A quasistatic evolution for a model in strain gradient plasticity. SIAM J. Math. Analysis, 40(3):1201-1245, 2008.

[44] P. Gudmundson. A unified treatment of strain gradient plasticity. J. Mech. Phys. Solids, 52:1379-1406, 2004.

[45] M.E. Gurtin. A gradient theory of single-crystal visco-plasticity that accounts for geometrically necessary dislocations. J. Mech. Phys. Solids, 50:5-32, 2002.

[46] M.E. Gurtin. A gradient theory of small deformation isotropic plasticity that accounts for the Burgers vector and for dissipation due to plastic spin. J. Mech. Phys. Solids, 52:2545-2568, 2004.

[47] M.E. Gurtin, A. Needleman. Boundary conditions in small-deformation, single-crystal plasticity that account for the Burgers vector. J. Mech. Phys. Solids. 53:1-31, 2005.

[48] M.E. Gurtin, L. Anand. A theory of strain gradient plasticity for isotropic, plastically irrotational materials. Part I: Small deformations. J. Mech. Phys. Solids, 53:1624-1649, 2005.

[49] M.E. Gurtin, L. Anand. A theory of strain gradient plasticity for isotropic, plastically irrotational materials. Part II: Finite deformation. Int. J. Plasticity, 21(12):2297-2318, 2005.

[50] M.E. Gurtin, L. Anand. Thermodynamics applied to gradient theories involving the accumulated plastic strain: The theories of Aifantis and Fleck and Hutchinson and their generalization. J. Mech. Phys. Solids, 57:405-421, 2009.

[51] M.E. Gurtin, E. Fried, L. Anand. The Mechanics and Thermodynamics of Continua. Cambridge University Press, Cambridge, 2010.

[52] M.E. Gurtin, B.D. Reddy. Gradient single-crystal plasticity within a von Mises-Hill framework based on a new formulation of self- and latent-hardening relations. J. Mech. Phys. Solids. 68:134-160, 2014.

[53] K. Hackl, Generalized standard media and variational principles in classical and finite strain elastoplasticity. J. Mech. Phys. Solids, 45:667-688, 1997.

[54] K. Hackl, F.D. Fischer. On the relation between the principle of maximum dissipation and inelastic evolution given by dissipation potentials. Proc. Roy. Soc. A, 464(2089):117-132, 2008.

[55] K. Hackl, S. Heinz, A. Mielke. A model for the evolution of laminates in finite-strain elastoplasticity. Zeit. f. Angew. Math. Mech., 92(11-12):888-909, 2012.

[56] K. Hackl, U. Hoppe, D.M. Kochmann, Variational modeling of microstructures in plasticity," in ed. J. Schröder, K. Hackl, Plasticity and Beyond. 65-129. Springer Vienna, 2014.

[57] K. Hackl, D.M. Kochmann. Relaxed potentials and evolution equations for inelastic microstructures, in ed. B.D. Reddy, IUTAM-Symposium on Theoretical, Modelling and Computational Aspects of Inelastic Media (in Cape Town, 2008). 27-39. Springer, Berlin, 2008.

[58] K. Hackl, F. Fischer, J. Svoboda. A study on the principle of maximum dissipation for coupled and noncoupled non-isothermal processes in materials. Proc. Roy. Soc. A, 467(2128):1186-1196, 2011.

[59] K. Hackl, F. Fischer, J. Svoboda. A study on the principle of maximum dissipation for coupled and noncoupled non-isothermal processes in materials, addendum. Proc. Roy. Soc. A, 467(2132):2422-2426, 2011.

[60] W. Han, B.D. Reddy. Plasticity: Mathematical Theory and Numerical Analysis. Springer-Verlag, New-York, 1999.

[61] Q.-C. He, C. Vallée, C. Lerintiu. Explicit expressions for the plastic normality-flow rule associated to the Tresca yield criterion. Z. Angew. Math. Phys., 56:357-366, 2005.

[62] R. Hill. The Mathematical Theory of Plasticity. Oxford University Press, New York, 1950. 
[63] R. Hill. A variational principle of maximum plastic work in classical plasticity. Quat. J. Mech. Appl. Math., 1(1):18-28, 1948.

[64] D.M. Kochmann, K. Hackl. The evolution of laminates in finite crystal plasticity: a variational approach. Cont. Mech. Thermod., 23:63-85, 2011.

[65] N. Kraynyukova, P. Neff, S. Nesenenko, K. Chełmiński. Well-posedness for dislocation based gradient viscoplasticity with isotropic hardening. Nonlinear Analysis: Real World Applications, 25:96-111, 2015.

[66] J. Krishnan, D.J. Steigmann. A polyconvex formulation of isotropic elastoplasticity. IMA Journ. Appl. Math., 79:722-738, 2014.

[67] E. Kröner. Continuum theory of defects. In: Les Houches, Session 35, 1980 - Physique des defauts, R. Balian et al. (Eds.). North-Holland, New York, pp. 215-315, 1981.

[68] M. Lazar. Dislocation theory as a 3-dimensional translation gauge theory, Ann. Phys. (Leipzig), 9:461-473, 2000.

[69] M. Lazar. An elastoplastic theory of dislocations as a physical field theory with torsion. J. Phys. A: Math. Gen., 35:1983-2004, 2002.

[70] M. Lazar and C. Anastassiadis. The gauge theory of dislocations: conservation and balance laws. Phil. Mag., 88:1673-1699, 2008.

[71] J. Lubliner. Plasticity Theory. Dover Publications, Mineola, New York, 2008.

[72] M. Lucchesi, M. Šilhavý. Il'yushin's conditions in non-isothermal plasticity. Arch. Ration. Mech. Anal.. 113:121-163, 1991.

[73] A. Mainik, A. Mielke. Existence results for energetic models for rate-independent systems. Calc. Var. Partial. Differ. Equ., 22(1):72-99, 2005.

[74] J. Mandel. Plasticité Classique et Viscoplasticité. Courses and Lectures, No 97, International Center for Mechanical Sciences, Udine (Berlin: Springer), 1971.

[75] J. Mandel. Equations constitutives et directeurs dans les milieux plastiques et viscoplasticques. Int. J. Solids Struct.. 9:725-740, 1973.

[76] J. Martin. Plasticity: Fundamental and general results. MIT Press, Cambridge, Mass., 1975.

[77] M. Menzel, P. Steinmann. On the formulation of higher gradient plasticity for single and polycrystals. J. Phys. France, 8:239-247, 1998.

[78] M. Menzel, P. Steinmann. On the continuum formulation of higher gradient plasticity for single and polycrystals. J. Mech. Phys. Solids, 48:1777-1796, 2000. Erratum: 49:1179-1180, 2001.

[79] A. Mielke. Analysis of energetic models for rate-independent materials. In T. Li, editor, Proceedings of the Int. Congress of Mathematicians 2002, Beijing, III: 817-828. Higher Education Press, 2002.

[80] A Mielke. Evolution of rate-independent systems. In. A. Dafermos and E. Feireisl, editors, Evolution equations. Vol. II, Handb. Differ. Equ., pages 461-559. Elsevier/North-Holland, Amsterdam, 2005.

[81] R. von Mises. Mechanik der plastischen Formänderung von Kristallen Zeit. Angew. Math. Mech., 8:161, 1928.

[82] J.J. Moreau. Application of convex analysis to the treatment of elastoplastic systems, in P. Germain and B. Nayroles, eds., Applications of Methods of Functional Analysis to Problems in Mechanics, Springer-Verlag, Berlin, 1976.

[83] H.B. Mühlhaus, E.C. Aifantis. A variational principle for gradient plasticity. Int. J. Solids Struct. 28(7):845853, 1991.

[84] P. Neff. On Korn's first inequality with non-constant coefficients Proc. Roy. Soc. Edinburgh Sect. A. 132(1):221-243, 2002.

[85] P. Neff. Remarks on invariant modelling in finite strain gradient plasticity. Technische Mechanik, 28(1):13-21, 2008.

[86] P. Neff. Uniqueness of strong solutions in infinitesimal perfect gradient plasticity. In ed. B.D. Reddy, IUTAMSymposium on Theoretical, Modelling and Computational Aspects of Inelastic Media (in Cape Town, 2008). 129-140. Springer, Berlin, 2008.

[87] P. Neff, I. Münch. Curl bounds Grad on SO(3). ESAIM Control Optim. Calc. Var. 14(1):148-159, 2008. 
[88] P. Neff, K. Chełmiński, H.D. Alber. Notes on strain gradient plasticity. Finite strain covariant modelling and global existence in the infinitesimal rate-independent case. Math. Mod. Meth. Appl. Sci., 19(2):1-40, 2009.

[89] P. Neff, I.-D. Ghiba, A. Madeo, L. Placidi, G. Rosi. A unifying perspective: the relaxed linear micromorphic continuum. Cont. Mech. Therm., 26:639-681, 2014.

[90] P. Neff, I.-D. Ghiba, M. Lazar, A. Madeo. The relaxed linear micromorphic continuum: well-posedness of the static problem and relations to the gauge theory of dislocations. Quart. J. Mech. Appl. Math.. 68:53-84, 2015.

[91] P. Neff, D. Pauly, K.J. Witsch. On a canonical extension of Korn's first and Poincaré's inequalities to H(Curl) motivated by gradient plasticity with plastic spin. Comp. Rend. Math. 349(23-24):1251-1254, 2011.

[92] P. Neff, D. Pauly, K.J. Witsch. On a canonical extension of Korn's first and Poincaré's inequalities to H(Curl). J. Math. Sci. (NY), 185(5):721-727, 2012.

[93] P. Neff, D. Pauly, K.J. Witsch. Maxwell meets Korn: A new coercive inequality for tensor fields with square integrable exterior derivatives. Math. Methods Applied Sciences. 35(1):65-71, 2012.

[94] P. Neff, D. Pauly, K.J. Witsch. Poincaré meets Korn via Maxwell: Extending Korn's first inequality to incompatible tensor fields. J. Diff. Equations. 258(4):1267-1302, 2014.

[95] P. Neff, A. Sydow, C. Wieners. Numerical approximation of incremental infinitesimal gradient plasticity. Int. J. Num. Meth. Engrg. 77(3):414-436, 2009.

[96] S. Nesenenko, P. Neff. Well-posedness for dislocation based gradient visco-plasticity I: Subdifferential case. SIAM J. Math. Anal.. 44(3):1695-1712, 2012.

[97] S. Nesenenko, P. Neff. Well-posedness for dislocation based gradient visco-plasticity II: General nonassociative monotone plastic flow. Math. Mech. Complex Systems. 1(2):149-176, 2013.

[98] Q.-S. Nguyen. Variational principles in the theory of gradient plasticity. C. R. Mecanique. 339:743-750, 2011.

[99] J.F. Nye. Some geometrical relations in dislocated solids. Acta Metall.. 1:153-162, 1953.

[100] E.T. Onat. The notion of state and its implications in thermodynamics of inelastic solids. Proc. of the IUTAM Symposium on irreversible aspects of the continuum mechanics and transfer of physical charateristics in moving fluids, Vienna. (Eds. H. Parkus and L.I. Sedov ), Springer-Verlag, Wien, 292-314, 1996.

[101] N. Ohno, D. Okumura. Higher-order stress and grain size effects due to self-energy of geometrically necessary dislocations. J. Mech. Phys. Solids, 55:1879-1898, 2007.

[102] M. Ortiz, E. Repetto. Nonconvex energy minimization and dislocation structures in ductile single crystals. J. Mech. Phys. Solids, 47(2):397-462, 1999.

[103] A. Panteghini, L. Bardella. On the finite element implementation of higher-order gradient plasticity, with focus on theories based on plastic distortion incompatibility. Comput. Methods Appl. Mech. Engrg., 310:840$865,2016$.

[104] A. Panteghini, L. Bardella. On the role of higher-order conditions in distortion gradient plasticity. J. Mech. Phys. Solids, 118:293-321, 2018.

[105] L.H. Poh. Scale transition of a higher order plasticity model - A consistent homogenization theory from meso to macro. J. Mech. Phys. Solids. 61:2692-2710, 2013.

[106] L.H. Poh, R.H.J. Peerlings, M.G.D. Geers, S. Swaddiwudhipong. An implicit tensorial gradient plasticity model - Formulation and comparison with a scalar gradient model. Int. J. Solids Struct., 48(18):2595-2604, 2011.

[107] L.H. Poh, R.H.J. Peerlings. The plastic rotation effect in an isotropic gradient plasticity model for applications at the meso scale. Int. J. Solids Struct., 78-79:57-69, 2016.

[108] C. Polizzotto. A link between the residual-based gradient plasticity theory and the analogous theories based on the virtual work principle. Int. J. Plasticity, 25:2169-2180, 2009.

[109] B.D. Reddy. The role of dissipation and defect energy in variational formulations of problems in straingradient plasticity. Part 1: Polycrystalline plasticity. Cont. Mech. Therm.. 23:527-549, 2011.

[110] B.D. Reddy. The role of dissipation and defect energy in variational formulations of problems in straingradient plasticity. Part 2: Single-crystal plasticity. Cont. Mech. Therm., 23:551-572, 2011. 
[111] B.D. Reddy, F. Ebobisse, A. McBride. Well-posedness of a model of strain gradient plasticity for plastically irrotational materials. Int. J. Plasticity, 24:55-73, 2008.

[112] B.D. Reddy, C. Wieners, B. Wohlmuth. Finite element analysis and algorithms for single-crystal straingradient plasticity. Int. J. Num. Meth. Engr. 90:784-804, 2014.

[113] M.B. Rubin. Physical reasons for abandoning plastic deformation measures in plasticity and visco-plasticity. Arch. Mech., 53:519-539, 2001.

[114] J.C. Simo, T.J.R. Hughes. Computational inelasticity. Singer-Verlag, New York, 1998.

[115] D.J. Steigmann, A. Gupta. Mechanically equivalent elastic-plastic deformations and the problem of plastic spin. Theoret. Appl. Mech. (Belgrade). 38:397-417, 2011.

[116] J.S. Stölken, A.G. Evans. A microbend test method for measuring the plasticity length scale. Acta Mater., 46:5109-5115, 1998.

[117] P.-M. Suquet. Sur un espace fonctionel pour les équations de la plasticité. Ann. Fac. Sci. Toulouse Math. (5), 1(1):77-87, 1979.

[118] P.-M. Suquet. Sur les équations de la plasticité: existence et regularité des solutions. J. Mécanique, 20:3-39, 1981.

[119] B. Svendsen. Continuum thermodynamic models for crystal plasticity including the effects of geometrically necessary dislocations. J. Mech. Phys. Solids. 50(25):1297-1329, 2002.

[120] B. Svendsen, P. Neff, A. Menzel. On constitutive and configurational aspects of models for gradient continua with microstructure. Z. Angew. Math. Mech., 89(8):687-697, 2009.

[121] H.E. Tresca. Mémoires sur l'écoulement des corps solides. Mém. Sav. Acad. Sci., Paris, (Sciences Mathématiques et physiques). 10:75-135, 1872.

[122] I. Tsagrakis, E.C. Aifantis. Recent developements in gradient plasticity - Part I: Formulation and size effects. J. Eng. Mater. Technol.. 124(3):352-357, 2002.

[123] I. Tsagrakis, G. Efremidis, A. Konstantinidis, E.C. Aifantis. Deformation vs. flow and wavelet-based models of gradient plasticity: Examples of axial symmetry. Int. J. Plasticity. 22:1456-1485, 2006.

[124] H.M. Zbib, E.C. Aifantis. On the gradient-dependent theory of plasticity and shear banding. Acta Mechanica. 92:209-225, 1992. 